HD-THEP-08-21

CPHT-RR075.0908

LPT-ORSAY-08-77

\title{
Non-Abelian bundles on heterotic non-compact K3 orbifold blowups
}

\author{
Stefan Groot Nibbelink ${ }^{a, 1}$, Filipe Paccetti Correia ${ }^{b, 2}$, Michele Trapletti $^{c, 3}$ \\ ${ }^{a}$ Institut für Theoretische Physik, Universität Heidelberg, Philosophenweg 16 und 19, D-69120 \\ Heidelberg, Germany \\ Shanghai Institute for Advanced Study, University of Science and Technology of China, 99 Xiupu Rd, \\ Pudong, Shanghai 201315, P.R. China \\ ${ }^{b}$ Centro de Física do Porto, Faculdade de Ciências da Universidade do Porto, \\ Rua do Campo Alegre, 687, 4169-007 Porto, Portugal \\ ${ }^{c}$ Laboratoire de Physique Theorique, Bat. 210, Université de Paris-Sud, F-91405 Orsay, France \\ Centre de Physique Théorique, École Polytechnique, F-91128 Palaiseau, France
}

\begin{abstract}
Instantons on Eguchi-Hanson spaces provide explicit examples of stable bundles on non-compact four dimensional $\mathbb{C}^{2} / \mathbb{Z}_{n}$ orbifold resolutions with non-Abelian structure groups. With this at hand, we can consider compactifications of ten dimensional $\mathrm{SO}(32)$ supergravity (arising as the low energy limit of the heterotic string) on the resolved spaces in the presence of non-Abelian bundles. We provide explicit examples in the resolved $\mathbb{C}^{2} / \mathbb{Z}_{3}$ case, and give a complete classification of all possible effective six dimensional models where the instantons are combined with Abelian gauge fluxes in order to fulfil the local Bianchi identity constraint. We compare these models with the corresponding $\mathbb{C}^{2} / \mathbb{Z}_{3}$ orbifold models, and find that all of these gauge backgrounds can be related to configurations of vacuum expectation values (VEV's) of twisted and sometimes untwisted states. Gauge groups and spectra are identical from both the orbifold and the smooth bundle perspectives.
\end{abstract}

\footnotetext{
E-mail: grootnib@thphys.uni-heidelberg.de

E-mail: paccetti@fc.up.pt

E-mail: michele.trapletti@cpht.polytechnique.fr
} 


\section{Introduction}

One of the central aims of string phenomenology is to construct models that are close relatives of the Standard Model (SM) or of its supersymmetric extension (MSSM). There have been many attempts in that direction, see e.g. [1-5], in this work we mainly focus on heterotic orbifold and Calabi-Yau constructions.

Orbifold compactification of the heterotic string [6-8] has been one of the most successful approaches to string phenomenology. One of its main advantages is that strings on orbifolds define exact CFTs and are therefore fully calculable. Many MSSM-like models have been constructed [9-11] following the route of building six dimensional intermediate "orbifold GUTs" [12] from string compactifications $[9,13-16]$. But this approach has the severe limitation that away from the orbifold point in moduli space one quickly looses control over the resulting effective theory. Moving away from the orbifold point is described by giving vacuum expectation values (VEV's) to some twisted states, which only makes sense when these vevs are sufficiently small, hence one does not have access to the full moduli space.

A generic point in the moduli space can only be described by giving the corresponding Calabi-Yau with a stable gauge bundle that it can support. This brings us to the second successful approach to obtain the MSSM from the heterotic string as a compactification on elliptically fibered Calabi-Yau manifolds with stable bundles $[17,18]$ on them [19-23]. These two procedures are very different, hence it is very difficult to decide whether they are closely related and give rise to the identical models. This might well be often the case because orbifolds are typically considered as singular limits of smooth Calabi-Yau spaces. It is this very interesting question, how these two approaches can be related to each other, that provides part of the inspiration for our work.

In recent publications we have made first attempts to understand the relation between heterotic string orbifold constructions and smooth Calabi-Yau manifolds with gauge bundles (see [24] for earlier work). To this end we have constructed explicit blowups of $\mathbb{C}^{n} / \mathbb{Z}_{n}$ orbifolds with Abelian gauge backgrounds satisfying the Hermitean Yang-Mills equations. We have shown that their gauge group and massless spectra precisely correspond to heterotic models built on these orbifolds [25] (see also [26]). Building on these results, we investigated the issue of multiple anomalous U(1)'s in blowup [27], and how these results can be extended to the study of compact orbifold blowups [28]. However, generically it is not easy to obtain explicit resolutions, but luckily techniques of toric geometry can be employed to resolve many much more complicated orbifold singularities $[29,30]$ and can even be lifted to describe the geometry of compact orbifold resolutions [31]. To be able to also study the relation between heterotic strings on such generic orbifolds and their toric resolutions, we constructed line bundles on them that characterize Abelian gauge backgrounds [32]. For essentially all the heterotic orbifold models we considered, we were able to find corresponding line bundle models, that have matching unbroken gauge groups and spectra (some exceptions are heterotic orbifolds without first twisted states, where no blow up is possible.) These analyses show that non-compact orbifold models with a single twisted field taking a non-vanishing VEV along a supersymmetric, i.e. F- and D-flat direction, that generates the blowup, can be matched with line bundle models with Abelian structure groups built on their toric resolutions.

However, line bundles only define a very small subclass of possible stable bundles on orbifold resolutions: There exist many other stable bundles that correspond to non-Abelian gauge backgrounds. This is also clear from the heterotic orbifold model perspective: Only a single of their twisted states takes a non-vanishing VEV to generate one of the line bundle models on the resolution. Clearly, 
there are other F- and D-flat directions in which multiple twisted and untwisted states take non-zero VEV's simultaneously. Therefore, a more complete understanding of the relation between orbifold models with VEV's switched on and non-Abelian bundle models is required.

In this work we take a first step in this direction by studying this issue for compactifications on noncompact K3 spaces preserving six dimensional $N=1$ supersymmetry. We consider Eguchi-Hanson resolutions [33-35] of the non-compact orbifolds $\mathbb{C}^{2} / \mathbb{Z}_{n}$, because, not only are these spaces known explicitly, but also a basis of all Abelian gauge configurations have been built on them. In addition even a large class of non-Abelian gauge backgrounds have been constructed in the past [36,37]. After we have reviewed the explicit constructions and discussed how these results can be described using a language inspired by toric geometry, we systematically classify all the possible resolutions with Abelian and non-Abelian backgrounds combined embedded in $\mathrm{SO}(32)$, that fulfill the local integrated Bianchi identity. (We focus here mainly for simplicity only on the ten dimensional $N=1 \mathrm{SO}(32)$ heterotic supergravity, the $\mathrm{E}_{8} \times \mathrm{E}_{8}$ can be treated similarly.) For each of these bundle models we are able to give the corresponding configuration of VEV's of twisted and untwisted states the heterotic $\mathrm{SO}(32)$ theory, that result in the same gauge group and six dimensional chiral spectrum. In this sense the present paper can be seen as the extension of the work [24] where this matching was established for line bundles only. For concreteness we perform most of this study for the resolution of the orbifold $\mathbb{C}^{2} / \mathbb{Z}_{3}$; we are confident that our results can be generalized to other $\mathbb{C}^{2} / \mathbb{Z}_{n}$ blowups as well.

\section{Eguchi-Hanson $\mathbb{C}^{2} / \mathbb{Z}_{N}$ resolutions}

In this section we give an explicit description of the resolution of $\mathbb{C}^{2} / \mathbb{Z}_{N}$ singularities using EguchiHanson spaces. After describing the geometry we first consider Abelian gauge backgrounds on these spaces, and then we turn to non-Abelian configurations realized as instantons. This subsection has been based to a large extend on [37] (see also [38]).

\subsection{Geometry}

The starting point of the description of Eguchi-Hanson spaces $[33,35,39]$ in four Euclidean dimensions is the line element

$$
\mathrm{d} s^{2}=V^{-1}\left(\mathrm{~d} x_{4}+\vec{\omega} \cdot \mathrm{d} \vec{x}\right)^{2}+V d \vec{x}^{2},
$$

or equivalently the vielbein one-forms:

$$
\vec{e}=V^{\frac{1}{2}} \mathrm{~d} \vec{x}, \quad e_{4}=V^{-\frac{1}{2}}\left(\mathrm{~d} x_{4}+\vec{\omega} \cdot \mathrm{d} \vec{x}\right) .
$$

Here we use the three dimensional vector notation $\vec{x}^{T}=\left(x_{1}, x_{2}, x_{3}\right) \in \mathbb{R}^{3}$, and make use of the standard vector inner and outer products. Instead, $x_{4}$ has compact range, that will be determined below. $V$ and $\vec{\omega}$ are scalar and vector functions of $\vec{x}$ only; we denote derivative w.r.t. $x_{i}, i=1,2,3$ as $V_{, i}$, etc. The spin-connection one-form is defined via the Maurer-Cartan structure equations

$$
\mathrm{d} e_{A}+\Omega_{A B} e_{B}=0, \quad \Omega_{A B}=-\Omega_{B A},
$$

where $A=1,2,3,4$. A short computation shows that the independent components read:

$$
\begin{aligned}
& \Omega_{4 i}=\frac{1}{2} V^{-\frac{3}{2}}\left\{-V_{, i} e_{4}-\left(\omega_{i, j}-\omega_{j, i}\right) e_{j}\right\}, \\
& \Omega_{i j}=\frac{1}{2} V^{-\frac{3}{2}}\left\{V_{, j} e_{i}-V_{, i} e_{j}+\left(\omega_{i, j}-\omega_{j, i}\right) e_{4}\right\} .
\end{aligned}
$$


The curvature two-form in turn is obtained via the conventional expression

$$
R_{A B}=\mathrm{d} \Omega_{A B}+\Omega_{A C} \Omega_{C B},
$$

The defining property of an Eguchi-Hanson space is that it has a self-dual curvature two-form

$$
R_{A B}=-\frac{1}{2} \epsilon_{A B C D} R_{C D}=* R_{A B} .
$$

Here $\epsilon_{A B C D}$ denotes the four dimensional epsilon tensor, with $\epsilon_{1234}=1$. The Hodge $*$-operation acts as

$$
*\left(e_{A} e_{B}\right)=-\frac{1}{2} \epsilon_{A B C D} e_{C} e_{D}, \quad *^{2}=\mathbb{1},
$$

i.e. $*\left(e_{4} e_{i}\right)=\frac{1}{2} \epsilon_{i j k} e_{j} e_{k}$, given the relation $\epsilon_{i j k}=\epsilon_{i j k 4}$ between the three and the four dimensional epsilon tensor.

A self-dual curvature is obtained automatically if the spin-connection one-form itself is self-dual, this is guaranteed if

$$
V_{, i}=-\epsilon_{i j k} \omega_{j, k} \Rightarrow V_{, i i}=0 .
$$

This means that $V$ is an harmonic function of $\vec{x}$. The precise expression for this harmonic function distinguishes between Eguchi-Hanson spaces and Kaluza-Klein monopoles: For the former the harmonic function takes the form

$$
V(\vec{x})=\sum_{r=1}^{N} \frac{R / 2}{\left|\vec{x}-\vec{x}_{r}\right|},
$$

where the points $\vec{x}_{r}$ denote the $N$ centers of the Eguchi-Hanson space, and $R$ sets the scale of the geometry. (Kaluza-Klein monopoles have a similar expansion but with an additional non-vanishing constant added.)

At the centers the function $V$ has singularities, but this does not necessarily imply that the geometry is singular. To see this we zoom in on one of the centers, which can be assumed to be located at the origin, so that we can ignore the other centers, i.e. $V \rightarrow R /(2 \varrho)$ with $\varrho=|\vec{x}|$. Using spherical coordinates,

$$
x_{1}=\rho \sin \theta \sin \phi, \quad x_{2}=\rho \sin \theta \cos \phi, \quad x_{3}=\rho \cos \theta,
$$

the line element for a single center can be written as

$$
\left.\mathrm{d} s^{2}\right|_{\text {single }}=V^{-1}\left(\mathrm{~d} x_{4}+\frac{1}{2} R(\cos \theta-1) \mathrm{d} \phi\right)^{2}+V\left(\mathrm{~d} \varrho^{2}+\varrho^{2} \mathrm{~d} \theta^{2}+\varrho^{2} \sin ^{2} \theta \mathrm{d} \phi^{2}\right),
$$

which means that we have chosen a gauge in which

$$
\vec{\omega}^{T}=\frac{R}{2 \varrho} \frac{1}{\varrho+x_{3}}\left(x_{2},-x_{1}, 0\right) .
$$

By introducing the complex coordinates

$$
z_{1}=\sqrt{2 R} \varrho^{\frac{1}{2}} \cos \left(\frac{1}{2} \theta\right) e^{i x_{4} / R}, \quad z_{2}=\sqrt{2 R} \varrho^{\frac{1}{2}} \sin \left(\frac{1}{2} \theta\right) e^{i\left(\phi-x_{4} / R\right)},
$$


one sees that the Eguchi-Hanson space with a single center is flat

$$
\left.\mathrm{d} s^{2}\right|_{\text {single }}=\left|\mathrm{d} z_{1}\right|^{2}+\left|\mathrm{d} z_{2}\right|^{2},
$$

everywhere except possibly at the origin. In order that the space is flat there as well, no deficit angle should be present, this implies that

$$
x_{4} \sim x_{4}+2 \pi R
$$

is periodic with a period of $2 \pi R$. Therefore, if we want that the Eguchi-Hanson space has no singularities, all centers have the same radius $R$, as given in (9).

If $n$ of the $N$ center of an Eguchi-Hanson space come close together a $\mathbb{Z}_{n}$ orbifold singularity arises. This can be easily seen by reviewing the above argument when $n$ centers are on top of each other: Indeed, the metric for this case is obtained by replacing $R$ by $n R$. Therefore, this substitution can be made in all of the consequent results, in particular the complex coordinates now become

$$
z_{1}=\sqrt{2 n R} \varrho^{\frac{1}{2}} \cos \left(\frac{1}{2} \theta\right) e^{i x_{4} /(n R)}, \quad z_{2}=\sqrt{2 n R} \varrho^{\frac{1}{2}} \sin \left(\frac{1}{2} \theta\right) e^{i\left(\phi-x_{4} /(n R)\right)},
$$

except in the periodicity (15) of $x_{4}$. Now, since the Eguchi-Hanson space is non-singular when all centers are away from each other, and this fixes (15), when $n$ centers are on top of each other the periodicity of $x_{4}$ leads to the following $\mathbb{C}^{2} / \mathbb{Z}_{n}$ orbifold identification

$$
\left(z_{1}, z_{2}\right) \rightarrow\left(z_{1}^{\prime}, z_{2}^{\prime}\right)=\left(e^{2 \pi i / n} z_{1}, e^{-2 \pi i / n} z_{2}\right) .
$$

The complex structure that we have introduced above for the Eguchi-Hanson space, with one or multiple centers on top of each other, is not unique. In fact any Eguchi-Hanson space can be equipped with three complex structures, or a hyper-Kähler structure. The three Kähler forms,

$$
J_{i}=\frac{1}{\sqrt{2}}\left(e_{4} e_{i}-\frac{1}{2} \epsilon_{i j k} e_{j} e_{k}\right)=\frac{1}{\sqrt{2}}(1-*) e_{4} e_{i},
$$

of the hyper-Kähler structure are anti-self-dual, and define a Clifford algebra

$$
* J_{i}=-J_{i}, \quad\left\{J_{i}, J_{j}\right\}=2 \delta_{i j} \mathrm{Vol},
$$

where $\mathrm{Vol}=e_{1} e_{2} e_{3} e_{4}$ is the volume form of the Eguchi-Hanson space.

\subsection{Abelian gauge backgrounds}

An important aspect is that an Eguchi-Hanson space supports regular Abelian gauge fluxes $\mathcal{F}_{r}=\mathrm{d} \mathcal{A}_{r}$, taken to be anti-Hermitean, that satisfy the Hermitean-Yang-Mills equations

$$
\mathcal{F}_{r} J_{i}=0
$$

for $i=1,2,3$ on a hyper-Kähler manifold. As becomes clear below, these field strengths are labeled by $r$, the center of the Eguchi-Hanson space. Because $J_{i}$ are anti-self-dual, these conditions are identically satisfied if $\mathcal{F}_{r}$ are self-dual, i.e. can be written as

$$
\mathcal{F}_{r}=i F_{r i}\left(e_{4} e_{i}+\frac{1}{2} \epsilon_{i j k} e_{j} e_{k}\right)
$$


for real functions $F_{r i}$ of $\vec{x}$. The closure of the field strength of an Abelian gauge field, $\mathrm{d} \mathcal{F}_{r}=0$, implies that $F_{r i}=F_{r, i}$ for some scalar functions $F_{r}$. The other components of the closure relations require these functions fulfill the equation

$$
\left[V F_{r}\right]_{, i i}=V F_{r, i i}+2 V_{, i} F_{r, i}=0 .
$$

The first equality is obtained by using that $V$ is harmonic. Hence we conclude that $V F_{r}$ is harmonic as well, and hence can be expanded in terms of harmonic functions $1 /|\vec{x}-\vec{y}|$ with constant $\vec{y}$, hence we have $F_{r}(\vec{x})=1 /(V(\vec{x})|\vec{x}-\vec{y}|)$. This means that unless $\vec{y}$ equals one of the positions of the centers of the Eguchi-Hanson space, the gauge background is singular. Therefore, we associate to each center $\vec{x}_{r}$ a gauge background

$$
\mathcal{F}_{r}=\frac{i}{R}\left(\frac{V_{r}}{V}\right)_{, i}\left(e_{4} e_{i}+\frac{1}{2} \epsilon_{i j k} e_{j} e_{k}\right), \quad \text { with } \quad V_{r}(\vec{x})=\frac{R / 2}{\left|\vec{x}-\vec{x}_{r}\right|} .
$$

This field strength is obtained from the gauge connection given by

$$
\mathcal{A}_{r}=-\frac{i}{R} V^{-\frac{1}{2}}\left[V_{r} e_{4}-\vec{\omega}_{r} \cdot \vec{e}\right]
$$

where $\vec{\omega}_{r}$ is defined from $V_{r}$ via the equation (8). The normalization of the gauge connections $\mathcal{A}_{r}$ above has been chosen such that the corresponding gauge field strengths $\mathcal{F}_{r}$ define an orthonormal basis of self-dual two forms $[40,41]$

$$
\int \frac{\mathcal{F}_{r} \mathcal{F}_{s}}{(2 \pi)^{2}}=-\delta_{r s}
$$

where the integral is performed over the whole Eguchi-Hanson space.

Because $V=\sum_{r} V_{r}$, it follows that $\sum_{r} \mathcal{F}_{r}=0$, i.e. only $N-1$ of these $N$ gauge backgrounds are independent. A basis of the independent gauge backgrounds can be defined by

$$
\tilde{\mathcal{F}}_{r}=\mathcal{F}_{r+1}-\mathcal{F}_{r}
$$

for $r=1, \ldots, N-1$. It follows immediately from (25), that the inner products of these two-forms $\mathcal{F}_{r}$ gives rise to the Cartan matrix $G$ of the $A_{N-1}$ algebra of $\mathrm{SU}(\mathrm{N})$ :

$$
\int \frac{\tilde{\mathcal{F}}_{r} \tilde{\mathcal{F}}_{s}}{(2 \pi)^{2}}=-G_{r s}
$$

Therefore the embedding of the Abelian gauge background in the gauge group $\mathrm{SO}(32)$ of the heterotic theory, is encoded by

$$
\mathcal{A}(\boldsymbol{\rho})=\rho^{T} \tilde{\mathcal{A}}, \quad \rho_{r}=\rho_{I r} H_{I},
$$

where $\boldsymbol{\rho}^{T}=\left(\rho_{1}, \ldots, \rho_{N-1}\right)$ is an Cartan algebra valued vector with $H_{I}$ the generators of the Cartan subalgebra. We often also view $\boldsymbol{\rho}$ as collection of $N-1$ vector $\rho_{r}$ with components $\rho_{r}^{I}$. 


\subsection{Non-Abelian gauge backgrounds}

Eguchi-Hanson spaces also support non-Abelian gauge backgrounds. The tangent bundle obviously defines an example of a non-Abelian gauge background on this space. In this section we would like to review how a large class of non-Abelian fluxes, or instantons, can be constructed explicitly. Such instantons are generalizations [37] of the 't Hooft instantons [42] on $\mathbb{R}^{4}$. We first consider SU(2) gauge background and then at the end of this subsection comment how to construct gauge backgrounds with other structure groups.

Consider a gauge connection one-form

$$
\mathcal{A}=i V^{-\frac{1}{2}}\left[A_{4} e_{4}+\vec{A} \cdot \vec{e}\right]
$$

which takes values in the $\mathrm{SU}(2)$ algebra generated by the Pauli-matrices $\sigma_{i}$. In order that the corresponding non-Abelian gauge field strength $\mathcal{F}=\mathrm{d} \mathcal{A}+\mathcal{A}^{2}$ satisfies the Hermitean-Yang-Mills equations (20), it has to be self-dual as the Abelian gauge backgrounds discussed in the previous subsection. This implies that the matrix-valued one-forms $A_{4}$ and $\vec{A}$ satisfy

$$
-A_{4, i}+i\left[A_{4}, A_{i}\right]=\epsilon_{i j k}\left(-A_{j, k}+\frac{i}{2}\left[A_{j}, A_{k}\right]\right) .
$$

To solve this equation we make the ansatz for the potential one-forms

$$
A_{4}=P_{i} \sigma_{i}, \quad A_{i}=-\epsilon_{i j k} P_{j} \sigma_{k},
$$

where $P_{i}$ are scalar functions to be determined. Substituting this ansatz into the equation above, leads to two independent relations

$$
P_{i, j}-P_{j, i}=0, \quad P_{i, i}-2\left(P_{i}\right)^{2}=0 .
$$

The first identity implies that $P_{i}=P_{, i}$ of a single scalar function $P$; the second equation implies that this can be expressed as

$$
P(\vec{x})=-\frac{1}{2} \ln H(\vec{x}),
$$

where $H$ is again an harmonic function. The centers of this harmonic function have to coincide with some of the centers of the Eguchi-Hanson space, otherwise the background is a configuration that does not have finite action, i.e. is singular. We will often say that the harmonic function $H$ and therefore the corresponding instanton are supported at some of the centers of the Eguchi-Hanson space. To summarize, the gauge background becomes

$$
\mathcal{A}=-i V^{-\frac{1}{2}}\left\{\frac{H_{, k}}{H} e_{4}+\epsilon_{i j k} \frac{H_{, i}}{H} e_{j}\right\} \frac{1}{2} \sigma_{k}=-V^{-\frac{1}{2}} \frac{H_{, A}}{H} e_{B} \frac{1}{2} \gamma_{A B}^{+}
$$

where after the second equal sign we have used the four component spinor notation of $\mathrm{SO}(4)$ to emphasize that the non-Abelian bundle only affects the positive chirality sector. (For our conventions concerning spinor representation properties see Appendix B.) Its field strength reads

$$
\mathcal{F}=\frac{i}{2} V^{-1}\left\{\left(\frac{H_{, i j}}{H}-2 \frac{H_{, i}}{H} \frac{H_{, j}}{H}-\frac{V_{, i}}{V} \frac{H_{, j}}{H}\right) \sigma_{j}+\frac{H_{, m}^{2}}{H^{2}} \sigma_{i}\right\}\left(e_{4} e_{i}+\frac{1}{2} \epsilon_{i k l} e_{k} e_{l}\right) .
$$


As a first important example of a non-Abelian gauge background, we consider the standard embedding in which the gauge connection is determined by the spin-connection

$$
\mathcal{A}_{S E}=\left(\Omega_{4 k}+\frac{1}{2} \Omega_{i j} \epsilon_{i j k}\right) \frac{i}{2} \sigma_{k} .
$$

By comparing the expressions for $\Omega_{4 i}$ and $\Omega_{i j}$ given in (4) and the generic non-Abelian gauge background (34), we infer that for the standard embedding we have $H(\vec{x})=V(\vec{x})$. Therefore the standard embedding is a non-Abelian gauge background that has support at all centers of the underlying Eguchi-Hanson space. Other non-Abelian gauge backgrounds are not supported at all Eguchi-Hanson centers.

The non-Abelian gauge backgrounds above are classified by their instanton numbers

$$
\int c_{2}(\mathcal{F})=\int \frac{1}{2} \operatorname{tr}\left(\frac{\mathcal{F}}{2 \pi i}\right)^{2}
$$

obtained as integrals over the second Chern class, for this see e.g. [43] (moreover, $c_{1}(\mathcal{F})=0$ ). The instanton number is related to the number $p$ of Eguchi-Hanson centers where a non-Abelian gauge flux has support. To determine this relation, we make the following observations: Away from the centers, the gauge configuration is pure gauge, hence the field strength vanishes there. Therefore, the only contributions to the instanton number come from the centers of the non-Abelian background and the asymptotic for $\vec{x} \rightarrow \infty$. To compute the contribution from the centers, we consider a small ball $B_{\vec{x}_{I}}$ surrounding the center $\vec{x}_{I}$, and we use Stoke's theorem

$$
\int_{B_{\vec{x}_{I}}} c_{2}(\mathcal{F})=\int_{\partial B_{\vec{x}_{I}}} \omega_{C S}(\mathcal{A})=\frac{1}{8 \pi^{2}} \int_{\partial B_{\vec{x}_{I}}} \frac{1}{3} \operatorname{tr} \mathcal{A}^{3}=1 .
$$

Here we used that only the second term of the Chern-Simons three-form $\omega_{C S}(\mathcal{A})=-\operatorname{tr}(\mathcal{F} \mathcal{A}-$ $\left.\frac{1}{3} \mathcal{A}^{3}\right) /\left(8 \pi^{2}\right)$ does not vanish. This computation holds for each center separately, when all centers are at finite distance from each other. Because this is a topological quantity even in the limit when $p$ centers come close together, each of them still has an instanton number 1 , hence collectively they have instanton number $p$. The instanton number at infinity can be computed in a similar way, but now only the leading contributions have to be taken into account. For an Eguchi-Hanson space with an instanton that is supported at $p$ of its $N$ centers this means that

$$
V(\vec{x})=\frac{N R}{2|\vec{x}|}, \quad H(\vec{x})=\frac{p R}{2|\vec{x}|},
$$

for large $|\vec{x}|$. Since $H$ only appears in a logarithm, that determines the non-Abelian gauge connection, the pre-factor in $H$ is in fact irrelevant. Hence, the integral over the region $X_{\varrho}=\{\vec{x},|\vec{x}|>\varrho\}$ gives, using Stoke's,

$$
\int_{X_{r}} c_{2}(\mathcal{F})=-\frac{1}{N}
$$

when $\varrho \rightarrow \infty$ because the orientation is opposite w.r.t. that around the centers of the instanton. Collecting the various contributions we conclude that the instanton number of an instanton with support at $p$ of its $N$ centers of a Eguchi-Hanson space is given by

$$
\int c_{2}(\mathcal{F})=p-\frac{1}{N} .
$$


The instantons discussed so far only define $\mathrm{SU}(2)$ gauge configurations, instantons in other gauge representations can be easily obtained from these. A complete and general investigation of instantons on Eguchi-Hanson spaces involves a combined ADHM [44,45] and Kronheimer-Nakajima [46-48] construction, for a comprehensive review see e.g. [37,49]. We make use of an easier but less general approach [50] (reviewed in [51]) in which the spin- $\frac{1}{2}$ generators $\frac{1}{2} \sigma_{i}$ of $\mathrm{SU}(2)$ are replaced by generators $T_{i}$ in a generic representation of $\mathrm{SU}(2)$ in the expressions for the gauge background (34). In particular the instanton number (37) in that representation is obtained by replacing $\operatorname{tr}\left(\frac{1}{2} \sigma_{i} \frac{1}{2} \sigma_{j}\right)$ by $\operatorname{tr}\left(T_{i} T_{j}\right)$. An irreducible representation $\mathbf{R}_{\mathbf{j}}$ is labeled by the spin quantum number $j=0, \frac{1}{2}, 1, \frac{3}{2}$, etc.; its dimension and quadratic Casimir are given by $\operatorname{dim} \mathbf{R}_{j}=2 j+1$ and $C_{j}=j(j+1)$ respectively. Therefore, the instant number of representation $\mathbf{R}_{j}$ is

$$
k_{j}=\frac{2}{3} C_{j} \operatorname{dim} \mathbf{R}_{j}=\frac{2}{3} j(j+1)(2 j+1)
$$

times larger than that in the fundamental spin $-\frac{1}{2}$ representation. If we embed a spin- $j$ representation in $\mathrm{SU}(\mathrm{M})$ with $M \geq 2 J+1$, a $\mathrm{SU}(2 j+1)$ subgroup is filled up, hence the subgroup $\mathrm{SU}(M-2 j-1)$ remains unbroken.

For the embedding of instanton configurations in $\mathrm{SO}(32)$ groups, which is of main interest in this paper on heterotic $\mathrm{SO}(32)$ blowup models, it is important to realize that $\mathrm{SO}(4)=\mathrm{SU}(2)_{+} \times \mathrm{SU}(2)_{-}$on the level of the algebra, where the \pm on the $\mathrm{SU}(2)$ s refer to the chiralities of the spinor representations. Explicit representations of the $\mathrm{SU}(2)_{ \pm}$are $\gamma_{A B}^{ \pm}$defined in Appendix B. Hence using the spin $-\frac{1}{2}$ configuration we the symmetry breaking pattern reads

$$
\mathrm{SO}(32) \rightarrow \mathrm{SO}(28) \times \mathrm{SU}(2)_{+} \times \mathrm{SU}(2)_{-} \rightarrow \mathrm{SO}(28) \times \mathrm{SU}(2)_{-},
$$

because the gauge background has positive chirality, see (34). When we consider the embedding of a second identical spin- $\frac{1}{2}$ instanton, the chirality forces us to embed it in the $\mathrm{SO}(28)$. The surviving gauge group in this case is $\mathrm{SO}(24) \times \mathrm{Sp}(4)_{-}$. The explicit representation of the generators of this symplectic group is given in Appendix B. Similarly, when we have a triple or quadruple embedding of identical instantons, we obtain the left-over symmetry groups $\mathrm{SO}(20) \times \mathrm{Sp}(6)_{-}$and $\mathrm{SO}(16) \times \mathrm{Sp}(8)_{-}$, respectively. Finally, it is possible to use the spin-1 embedding into $\mathrm{SO}(32)$, because this representation is a vector representation, it induces the symmetry breaking to $\mathrm{SO}(29)$.

\section{Toric $\mathbb{C}^{2} / \mathbb{Z}_{N}$ resolutions}

We review the resolution $\operatorname{Res}\left(\mathbb{C}^{2} / \mathbb{Z}_{N}\right)$ described using toric geometrical terms, and give a systematic account of gauge fluxes on such resolutions. This section is based in part on [31,32]. (For a more detailed account on toric geometry, see e.g. [52-54].)

\subsection{Geometry}

Let $z_{1}, z_{2}$ denote the coordinates of $\mathbb{C}^{2}$ associated with those of the orbifold $\mathbb{C}^{2} / \mathbb{Z}_{N}$ before the blowup, and $x_{1}, \ldots x_{r}, r=1, \ldots N-1$ the additional homogeneous coordinates that define the toric variety

$$
\operatorname{Res}\left(\mathbb{C}^{2} / \mathbb{Z}_{N}\right)=\left(\mathbb{C}^{N+1}-\{0\}\right) /\left(\mathbb{C}^{*}\right)^{N-1}
$$


The extra homogeneous coordinates $x_{r}$ are associated with the twisted sectors $w_{r}=(r, N-r) / N$ of a $\mathbb{C}^{2} / \mathbb{Z}_{N}$ orbifold theory. The local coordinates constructed from the homogeneous ones

$$
Z_{1}=z_{1} \prod_{r=1}^{N-1} x_{r}^{(N-r) / N}, \quad Z_{2}=z_{2} \prod_{r=1}^{N-1} x_{r}^{r / N},
$$

are invariant under the complex scalings:

$$
\begin{aligned}
\left(z_{1}, x_{1}, x_{2}\right) & \sim\left(\lambda_{1} z_{1}, \lambda_{1}^{-2} x_{1}, \lambda_{1} x_{2}\right) \\
& \vdots \\
\left(x_{N-2}, x_{N-1}, z_{2}\right) & \sim\left(\lambda_{N-1} x_{N-2}, \lambda_{N-1}^{-2} x_{N-1}, \lambda_{N-1} z_{2}\right),
\end{aligned}
$$

where $\lambda_{1}, \ldots, \lambda_{N-1} \in \mathbb{C}^{*}$.

The ordinary and exceptional divisors are defined as $D_{i}=\left\{z_{i}=0\right\}, i=1,2$, and $E_{r}=\left\{x_{r}=0\right\}$, $r=1, \ldots, N-1$, respectively. The exceptional divisors are compact, while the ordinary ones are not. From the fan of the toric diagram we read off the intersections

$$
E_{r} E_{r+1}=1
$$

for $r=0, \ldots N$, when we write $E_{0}=D_{2}$ and $E_{N}=D_{1}$. The self-intersections of the exceptional divisors equal

$$
E_{r}^{2}=-2,
$$

with $r=1, \ldots, N-1$. The intersections of the exceptional divisors can be conveniently grouped together as:

$$
\mathbf{E} \mathbf{E}^{T}=-G
$$

where $G=G\left(A_{N-1}\right)$ is the Cartan matrix of $\mathrm{SU}(\mathrm{N})$ and $\mathbf{E}^{T}=\left(E_{1}, \ldots, E_{N-1}\right)$. The ordinary divisors are not independent from the exceptional ones because of the following linear equivalence relations

$$
D_{1} \sim-\sum_{r=1}^{N-1} \frac{r}{N} E_{r}, \quad D_{2} \sim-\sum_{r=1}^{N-1} \frac{N-r}{N} E_{r} .
$$

These relations are compatible with the (self-)intersections given above, and can be used to show that the Euler number of the resolution is given by

$$
\chi\left(\operatorname{Res}\left(\mathbb{C}^{2} / \mathbb{Z}_{N}\right)\right)=\int c_{2}\left(\operatorname{Res}\left(\mathbb{C}^{2} / \mathbb{Z}_{N}\right)\right)=N-\frac{1}{N} .
$$

To obtain this one may expand to second order the total Chern class represented as a product over all divisors

$$
c\left(\operatorname{Res}\left(\mathbb{C}^{2} / \mathbb{Z}_{N}\right)\right)=\left(1+D_{1}\right)\left(1+D_{2}\right) \prod_{r=1}^{N-1}\left(1+E_{r}\right),
$$

and use the intersection numbers are described above. If one expands the total Chern class to first order and uses the linear equivalence relations (50), one finds zero. This shows that the space has vanishing first Chern class, i.e. a non-compact four dimensional Calabi-Yau. 


\subsection{Abelian gauge fluxes}

Next we turn to describe Abelian gauge configurations on the resolution of the $\mathbb{C}^{2} / \mathbb{Z}_{N}$ singularity. As an Abelian gauge flux $\mathcal{F}$ can be expanded in terms of the exceptional divisors, we may write

$$
\frac{\mathcal{F}}{2 \pi}=\rho^{T} \mathbf{E}=\rho_{1} E_{1}+\ldots+\rho_{N-1} E_{N-1},
$$

for some coefficients $\rho_{r}$ inside the vector $\boldsymbol{\rho}^{T}=\left(\rho_{1}, \ldots, \rho_{N-1}\right)$. These coefficients have to be chosen such that the gauge flux is properly quantized. This means that the entries of the vector

$$
\mathrm{Q}=-\int \mathbf{E} \frac{\mathcal{F}}{2 \pi}=G \boldsymbol{\rho},
$$

are all "charges", i.e. elements $Q_{r} \in \Lambda$, of the lattice spanned by vectorial and spinorial weights of $\mathrm{SO}(32)$. Any choice of the charges constitutes a valid gauge background $\frac{\mathcal{F}}{2 \pi}=\mathbf{Q}^{T} G^{-1} \mathbf{E}$, resulting in a contribution to the Bianchi identity

$$
-\frac{1}{2} \int\left(\frac{\mathcal{F}}{2 \pi}\right)^{2}=\frac{1}{2} \boldsymbol{\rho}^{T} G \boldsymbol{\rho}=\frac{1}{2} \mathbf{Q}^{T} G^{-1} \mathbf{Q} .
$$

On the resolution, the orbifold gauge shift vector $v$ can be computed as the flux around one of the coordinate axes, i.e. integrals over the divisors $D_{i}$. This identification has to hold only up to vectors out of the lattice $\Lambda$, denoted by "三”. Because the orientation of the orbifold action on the coordinates $z_{1}$ and $z_{2}$ is opposite, we have

$$
-v \equiv \int_{D_{2}} \frac{\mathcal{F}}{2 \pi}=\rho_{1}=\frac{1}{N} \sum_{r=1}^{N-1} r Q_{p-r}, \quad v \equiv \int_{D_{1}} \frac{\mathcal{F}}{2 \pi}=\rho_{N-1}=\frac{1}{N} \sum_{r=1}^{N-1}(N-r) Q_{p-r} .
$$

Either of these equations tells us that $v$ is properly quantized in units of $1 / N$, and that they are compatible because

$$
\rho_{1}+\rho_{N-1}=\sum_{r=1}^{N-1} Q_{r} \in \Lambda
$$

equals a lattice vector in any case. Therefore any choice of charges $\mathbf{Q}$ defines a consistent gauge background that can be identified with orbifold boundary conditions in the blow down limit.

To find the properly quantized $\rho$ is not so straightforward in general. Since in the latter part of this paper we focus on models on the resolution of $\mathbb{C}^{2} / \mathbb{Z}_{3}$ we remind the reader of the properly quantized bases found previously [32]

$$
\frac{\mathcal{F}_{V}}{2 \pi}=\left(V_{1}^{I} D_{1}+V_{2}^{I} D_{2}\right) H_{I}
$$

where $V_{1}$ and $V_{2}$ are vectorial or spinoral lattice vectors. Upon converting the $D$ 's to the E's and using the linear equivalence relations, we see that this means that

$$
\rho_{1}=-\frac{1}{3}\left(V_{1}+2 V_{2}\right), \quad \rho_{2}=-\frac{1}{3}\left(2 V_{1}+V_{2}\right) .
$$

The contribution to the Bianchi identity then reads

$$
-\frac{1}{2} \int \operatorname{tr}\left(\frac{\mathcal{F}_{V}}{2 \pi}\right)^{2}=\frac{1}{3}\left(V_{1}^{2}+V_{2}^{2}+V_{1} \cdot V_{2}\right) .
$$




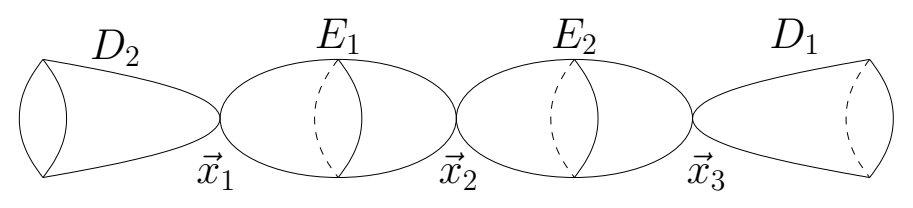

Figure 1: Schematic picture of the compact and non-compact curves within the resolution of $\mathbb{C}^{2} / \mathbb{Z}_{3}$ corresponding to the exceptional divisors $E_{r}$ and the ordinary divisors $D_{i}$, respectively.

\subsection{Relation with explicit construction of (non-)Abelian gauge fluxes}

In the previous section we have discussed explicit solutions of the non-compact Calabi-Yau condition and presented explicit constructions of Abelian and non-Abelian gauge backgrounds. Comparing the results of the Abelian gauge fluxes and the construction of the divisors shows, that we can make identifications between the exceptional and ordinary divisors and the characteristic classes corresponding to the gauge field strength (denoted by $[\ldots]$ )

$$
2 \pi E_{r}=\left[\tilde{\mathcal{F}}_{r}\right]=\left[\mathcal{F}_{r}-\mathcal{F}_{r+1}\right], \quad 2 \pi D_{1}=\left[\mathcal{F}_{N}\right] . \quad 2 \pi D_{2}=-\left[\mathcal{F}_{1}\right] .
$$

By the Poincaré duality we know that the divisors also have an interpretation as complex curves in the resolution space. For this we assume that all the centers $\vec{x}_{r}, r=1, \ldots N-1$, lie ordered on one line. The representation of the exceptional divisors are two-spheres suspended at two adjacent centers [37]

$$
E_{r}=\left\{\left(\vec{x}, x_{4}\right) \mid x_{4} \in\left[0,2 \pi R\left[, \vec{x}=\vec{x}_{r}+\lambda\left(\vec{x}_{r+1}-\vec{x}_{r}\right), \lambda \in[0,1]\right\} .\right.\right.
$$

Clearly these surfaces are compact, and only nearest neighbor divisors have non-vanishing intersection number one, as they intersect only at a single point: the center that they both have in common. In a similar way we can also give a representation of the non-compact ordinary divisors

$$
\begin{aligned}
& D_{1}=\left\{\left(\vec{x}, x_{4}\right) \mid x_{4} \in\left[0,2 \pi R\left[, \quad \vec{x}=\vec{x}_{N}+\lambda \vec{e}_{3}, \lambda \geq 0\right\},\right.\right. \\
& D_{2}=\left\{\left(\vec{x}, x_{4}\right) \mid x_{4} \in\left[0,2 \pi R\left[, \quad \vec{x}=\vec{x}_{1}-\lambda \vec{e}_{3}, \lambda \geq 0\right\},\right.\right.
\end{aligned}
$$

Hence, the intersections $D_{1} E_{N-1}=D_{2} E_{1}=1$ are consistent with what we found before. The Abelian gauge fluxes are thus associated with the complex curves between two centers of an Eguchi-Hanson space A schematic picture of these curves and their intersections is sketched in Figure 1.

Non-Abelian bundles on Eguchi-Hanson spaces we can describe by similar pictures. As we have seen in Subsection 2.3, instantons on Eguchi-Hanson spaces are supported at one or more centers of the Eguchi-Hanson space. In particular, the standard embedding is supported on all centers, and therefore all divisors participate to the total Chern class (52): Precisely because the standard embedding instanton is supported at each of the centers we cannot deform the curves at these points.

Instead, for the instanton $I_{\vec{x}_{2}}$ supported only at $\vec{x}_{2}$, we can merge the curves $D_{2}$ and $E_{1}$, because there is no obstruction created by the instanton. The resulting curve is denoted as $D_{2}+E_{1}$. Similarly the curves $D_{1}$ and $E_{2}$ can be merged to form $D_{1}+E_{2}$. This process is depicted in Figure 2 for the $\mathbb{C}^{2} / \mathbb{Z}_{3}$ singularity given in Figure 1 . Therefore, as far as the instanton supported only at $\vec{x}_{2}$ is concerned, there are only two divisors $D_{2}+E_{1}$ and $D_{1}+E_{2}$ relevant, and consequently its total Chern class reads

$$
c\left(I_{\vec{x}_{2}}\right)=\left(1+D_{2}+E_{1}\right)\left(1+D_{1}+E_{2}\right) .
$$




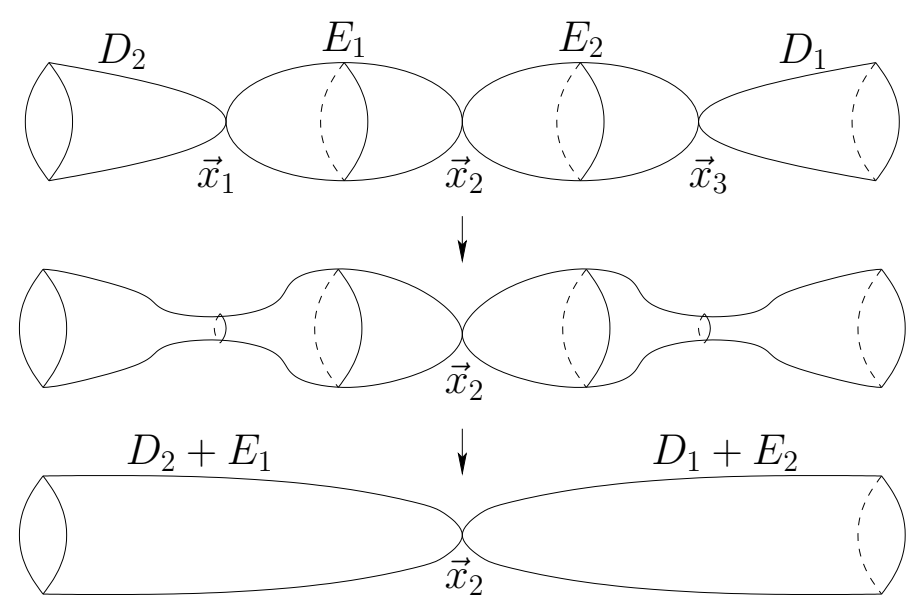

Figure 2: The two curves $D_{2}, E_{1}$ and $D_{1}, E_{2}$ are merged to form the curves $D_{2}+E_{1}$ and $D_{1}+E_{2}$, respectively.

Because this describes an $\mathrm{SU}(2)$ (non-Abelian) flux the first Chern class vanishes identically, as follows directly from expanding this to first order and using the linear equivalence relations (50). For the second Chern class we find

$$
\int c_{2}\left(I_{\vec{x}_{2}}\right)=1-\frac{1}{N}
$$

using the intersection numbers given above. This is consistent with the result computed in (41) using the explicit instanton solution on the Eguchi-Hanson space. One can check that also for instantons supported at multiple centers this procedure gives the correct value $p-1 / N$ for the second Chern class, and that this result only depends on the number $p$ of centers present in the instanton, not at their location.

\section{Blowup models on non-compact K3 orbifolds}

In the previous two sections we used both explicit constructions and implicit toric geometry methods to describe the geometry of non-compact resolutions of $\mathbb{C}^{2} / \mathbb{Z}_{N}$ orbifolds, and the Abelian and nonAbelian gauge configurations they can support. The purpose of this section is to show that the resulting models can be understood as non-compact heterotic orbifold models with certain VEV's switched on. For concreteness we restrict ourselves to models on the $\mathbb{C}^{2} / \mathbb{Z}_{3}$ orbifold only. The corresponding heterotic orbifold models are listed in Table 1. Below we list the possible smooth models obtained by combining the Abelian and non-Abelian bundles constructed in the previous sections. Since by definition all these configurations are supersymmetric as the gauge backgrounds were required to satisfy the Hermitian Yang-Mills equations, we restrict ourself to the blow-ups of heterotic $\mathbb{C}^{2} / \mathbb{Z}_{3}$ orbifold models that do not break supersymmetry, thus, we only consider VEV's along flat directions of the potential.

We stress the fact that the models we consider are non-compact, but they are built in a way such that compact (global) orbifolds can be recovered in the simplest possible way. In particular, we 


\begin{tabular}{|c|c|c|c|}
\hline$\#$ & $\begin{array}{l}\text { Gauge group } \\
\text { Shift vector }\end{array}$ & $\begin{array}{l}\text { Untwisted } \\
\text { matter }\end{array}$ & $\begin{array}{l}\text { Twisted } \\
\text { matter }\end{array}$ \\
\hline $3 a$ & $\begin{array}{c}S O(28) \times S U(2) \times U(1) \\
\frac{1}{3}\left(1^{2}, 0^{14}\right) \\
\end{array}$ & 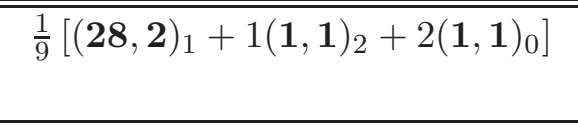 & $\begin{array}{c}(\mathbf{2 8}, \mathbf{2})_{-1 / 3}+5(\mathbf{1}, \mathbf{1})_{2 / 3} \\
+2(\mathbf{1}, \mathbf{1})_{4 / 3} \\
\end{array}$ \\
\hline $3 \mathrm{~b}$ & $\begin{array}{c}S O(22) \times S U(5) \times U(1) \\
\frac{1}{3}\left(1^{4}, 2,0^{11}\right)\end{array}$ & $\frac{1}{9}\left[(\mathbf{2 2}, \mathbf{5})_{1}+(\mathbf{1}, \mathbf{1 0})_{2}+2(\mathbf{1}, \mathbf{1})_{0}\right]$ & $\begin{array}{c}(\mathbf{2 2}, \mathbf{1})_{5 / 3}+(\mathbf{1}, \mathbf{1 0})_{-4 / 3} \\
+2(\mathbf{1}, \mathbf{5})_{-2 / 3}\end{array}$ \\
\hline $3 \mathrm{c}$ & $\begin{array}{c}S O(16) \times S U(8) \times U(1) \\
\frac{1}{3}\left(1^{8}, 0^{8}\right)\end{array}$ & $\frac{1}{9}\left[(\mathbf{1 6}, \mathbf{8})_{1}+(\mathbf{1}, \mathbf{2 8})_{2}+2(\mathbf{1}, \mathbf{1})_{0}\right]$ & $(\mathbf{1}, \mathbf{2 8})_{-2 / 3}+2(\mathbf{1}, \mathbf{1})_{8 / 3}$ \\
\hline $3 \mathrm{~d}$ & $\begin{array}{c}S O(10) \times S U(11) \times U(1) \\
\frac{1}{3}\left(1^{10}, 2,0^{5}\right)\end{array}$ & $\frac{1}{9}\left[(\mathbf{1 0}, \mathbf{1 1})_{1}+(\mathbf{1}, \mathbf{5 5})_{2}+2(\mathbf{1}, \mathbf{1})_{0}\right]$ & $(\mathbf{1}, \mathbf{1 1})_{-8 / 3}+(\overline{\mathbf{1 6}}, \mathbf{1})_{-11 / 6}$ \\
\hline $3 \mathrm{e}$ & $\begin{array}{c}S U(14) \times S U(2)^{2} \times U(1) \\
\frac{1}{3}\left(1^{14}, 0^{2}\right)\end{array}$ & $\frac{1}{9}\left[(\mathbf{1 4}, \mathbf{2}, \mathbf{2})_{1}+(\mathbf{9 1}, \mathbf{1}, \mathbf{1})_{2}+2(\mathbf{1})_{0}\right]$ & $\begin{array}{c}(\mathbf{1})_{14 / 3}+(\mathbf{1 4}, \mathbf{2}, \mathbf{1})_{-4 / 3} \\
+2(\mathbf{1}, \mathbf{1}, \mathbf{2})_{-7 / 3}\end{array}$ \\
\hline
\end{tabular}

Table 1: $\mathrm{SO}(32)$ heterotic orbifold spectra on $\mathbb{C}^{2} / \mathbb{Z}_{3}$, see e.g. $[24,55,56]$.

enforce on the local models all the conditions required in the global models, in this way we have that the spectrum of $T^{4} / \mathbb{Z}_{3}$ models can be obtained by just trivially summing over its $9 \mathbb{C}^{2} / \mathbb{Z}_{3}$ singularities, i.e. by multiplying by 9 the $\mathbb{C}^{2} / \mathbb{Z}_{3}$ spectra given in Table 1 .

order that a spectrum

\subsection{Abelian and non-abelian bundles on the resolved $\mathbb{C}^{2} / \mathbb{Z}_{3}$ singularity}

We consider a smooth resolution of the $\mathbb{C}^{2} / \mathbb{Z}_{3}$ orbifold. The Abelian and non-Abelian gauge configurations were discussed at length in subsections 2.2, 2.3 and 3.2, 3.3 as explicit and toric geometrical constructions, respectively. Their collective characterization can be summarized as follows: The vectors $V_{1}$ and $V_{2}$ define the embedding of the two line bundles present in the resolution, see (58). The number $n_{p}^{1 / 2}$ counts the number of $\mathrm{SU}(2)$ bundles embedded in $\mathrm{SO}(32)$ supported at $p$ centers on the Eguchi-Hanson resolution space ( $p=1,2,3$ because we treat the $\mathbb{Z}_{3}$ case). Finally $n_{p}^{1}$ is defined similar to $n_{p}^{1 / 2}$, but the spin-one representation of $\mathrm{SU}(2)$ is used instead.

To obtain non-compact resolution models, for which we are readily able to compute spectra, we enforce the local Bianchi identity

$$
\frac{1}{3}\left(V_{1}^{2}+V_{2}^{2}+V_{1} \cdot V_{2}\right)+\frac{4}{3} n_{1}^{1}+\frac{2}{3} n_{1}^{1 / 2}+\frac{5}{3} n_{2}^{1 / 2}+\frac{8}{3} n_{3}^{1 / 2}=K, \quad K=\frac{8}{3} .
$$

Other possible bundles could be present if we did not require the local Bianchi identity to be fulfilled, i.e. when $K \neq 8 / 3$. (The Bianchi-identity here is given in the normalization appropriate for SUgroups. The spin-1 embedding of the instanton is defined in $\mathrm{SO}(3)$, hence the factor in front of $n_{1}^{1}$ is $4 / 3$ rather than $4 * 2 / 3$. The relevant integrals are summarized in Table 2.) Since the contributions involving $V_{1}$ and $V_{2}$ always give a non-negative contribution, the instanton numbers of the (non)Abelian configurations $n=\left(n_{1}^{1}, n_{1}^{1 / 2}, n_{2}^{1 / 2}, n_{3}^{1 / 2}\right)$ satisfy $4 n_{1}^{1}+2 n_{1}^{1 / 2}+5 n_{2}^{1 / 2}+8 n_{3}^{1 / 2} \leq 8$. The possible configurations are listed in Table 3 . If this sum equals eight, only non-Abelian bundles are involved; 


\begin{tabular}{|c|c|c|c|}
\hline \multicolumn{2}{|c|}{$\operatorname{spin}-\frac{1}{2} \quad \mathrm{SU}(2)_{+}$} & \multicolumn{2}{|c|}{ spin-1 $\quad \mathrm{SO}(3)$} \\
\hline $\mathbf{r}_{\kappa}$ & $\frac{1}{8 \pi^{2}} \int \operatorname{tr}_{\mathbf{r}_{\kappa}}\left(\mathcal{F}^{\prime}\right)^{2}$ & $\mathbf{r}_{\kappa}$ & $\frac{1}{8 \pi^{2}} \int \operatorname{tr}_{\mathbf{r}_{\kappa}}\left(\mathcal{F}^{\prime}\right)^{2}$ \\
\hline 1 & 0 & 1 & 0 \\
\hline 2 & $p-\frac{1}{3}$ & 3 & $4\left(p-\frac{1}{3}\right)$ \\
\hline 3 & $4\left(p-\frac{1}{3}\right)$ & & \\
\hline
\end{tabular}

Table 2: Depending on the representation under the $\mathrm{SU}(2)$ or $\mathrm{SO}(3)$ group characterizing the embedding of the instantons the multiplicities of zero modes in six dimensions change. Finally, $p$ specifies on how many centers the instanton background is located.

if it vanishes only line bundles are employed; otherwise a mixture of both types is required. The identification of the line bundle vectors with the $\mathbb{Z}_{3}$ orbifold shift imposes that $V_{1} / 3=-V_{2} / 3$ up to the addition of lattice vectors [32]. In fact in most cases no lattice vectors are needed, thus, in general we have $V_{1}=-V_{2}=\left(0^{16-m_{1}-m_{2}}, 1^{m_{1}}, 2^{m_{2}}\right)$ with $m_{1}+m_{2}<16$, or $V_{1}=-V_{2}=\frac{1}{2}\left(1^{m_{1}}, 3^{m_{2}}\right)$ and $m_{1}+m_{2}=16$. One additional constraint is that the line bundle vectors are properly quantized such that the Freed-Witten anomaly $[57,58]$ does not arise: The first Chern class of the bundle, i.e. the sum of the entries of the line bundle vectors, needs to be even. From equation (66) it follows immediately, that if $n_{2}^{1 / 2}=1$ (or odd in general) then this condition is violated. Finding the relevant $m_{1}$ and $m_{2}$ is straightforward and the results are listed in the second column of Table 3.

Given the topological characterization of the Abelian and non-Abelian bundles, the gauge symmetry breaking they induce can be investigated. When the Abelian and non-Abelian gauge fluxes are embedded in different parts of $\mathrm{SO}(32)$, the resulting unbroken gauge group is the intersection of the groups that are unbroken by either flux. The other possibility is that the Abelian and the non-Abelian gauge backgrounds share some Cartan generators; they "overlap". Because the two types of fluxes commute with each other, if the $\mathrm{SU}(2)$ instanton has a Cartan generator, say $H_{1}+H_{2}$, and nonAbelian generators corresponding to the weights $\pm\left(1^{2}, 0^{14}\right)$, then the Abelian flux has to be embedded in the $\mathrm{SO}(32)$ Cartan as $H_{1}-H_{2}$. This Cartan "overlapping" of the non-Abelian and Abelian gauge flux has two consequences: The unbroken $\mathrm{SO}(N)$ group will be larger, while the $\mathrm{Sp}(2 n)$ or $\mathrm{SU}(2)$ group are (partially) broken. The amount of Cartan "overlap" of the Abelian flux with the instanton gauge configuration is indicated in the overbraced part of the line bundle vector $V_{1}$. The resulting unbroken gauge group is listed in the third column of Table 3. We stress that a specific overlap, treated in [59], is the case of a $U(2)$ bundle embedded in $\mathrm{SO}(32)$.

Once the gauge bundle has been topologically characterized, its embedding as a subgroup $H \in$ $\mathrm{SO}(32)$ gauge group has been specified, and the resulting unbroken gauge group $G$ has been determined, we can compute the full spectrum using index theorems or equivalently from the anomaly polynomial of the ten dimensional gaugino. For this we need to specify the branching of the adjoint representation into a sum of tensor product representations as

$$
\mathbf{A d}=496=\bigoplus_{\kappa}\left(\mathbf{r}_{\kappa}, \mathbf{R}_{\kappa}\right)
$$


where $\mathbf{r}_{\kappa}$ and $\mathbf{R}_{\kappa}$ denote irreducible representations of the non-Abelian part of $H$ and $G$, respectively. Under the assumption $V_{1}=-V_{2}=V$, we find that the multiplicity $N_{\kappa}$ of a state $\mathbf{R}_{\kappa}$ is determined by

$$
N_{\kappa}=\frac{1}{2} \frac{1}{(2 \pi)^{2}} \int\left\{\frac{1}{2} \operatorname{tr}_{\mathbf{r}_{\kappa}}\left(\mathcal{F}^{\prime}\right)^{2}+\frac{1}{2} \operatorname{dim} \mathbf{r}_{\kappa}\left(\left.\mathcal{F}_{V}^{2}\right|_{\mathbf{R}_{\kappa}}-\frac{1}{12} \operatorname{tr} \mathcal{R}^{2}\right)\right\}
$$

where $\mathcal{F}^{\prime}$ denotes the non-Abelian instanton background, $\mathcal{F}_{V}$ the Abelian gauge flux, and $\mathcal{R}$ the $\mathrm{SU}(2)$ curvature two-form. The integrals over the curvature and the U(1) background follow directly from the results of earlier parts of this paper, i.e.

$$
\frac{1}{8 \pi^{2}} \int \operatorname{tr} \mathcal{R}^{2}=\frac{8}{3}, \quad \frac{1}{8 \pi^{2}} \int \operatorname{tr} \mathcal{F}_{V}^{2}=\frac{1}{3} H_{V}^{2},
$$

where we denote by $H_{V}=V_{I} H_{I}$ the Cartan generator of the Abelian-bundle. The value of the operator $H_{V}^{2}$ has to be evaluated on each of the irreducible representations $\mathbf{R}_{\kappa}$ as the multiplicity number (68) indicates. More care needs to be taken when computing the integral over the nonAbelian instanton background $\mathcal{F}^{\prime}$, as it also depends on over which representation $\mathbf{r}_{\kappa}$ the trace is taken. For the spin- $\frac{1}{2}$ instantons this can be the singlet $\mathbf{1}$, the fundamental $\mathbf{2}$, or the adjoint $\mathbf{3}$, representations of $\mathrm{SU}(2)$; for the spin-1 instantons only the singlet or triplet representations of $\mathrm{SO}(3)$ are relevant for our purposes. In the cases where there are multiple non-Abelian instantons embedded, also traces over product representations occur. The basic values of the possible instanton numbers have been collected in table 2 .

The resulting spectra are given in the last column of Table 3. The computation of these spectra requires mostly standard group theory, see e.g. [60]. As only the representation theory of the $\operatorname{Sp}(2 n)$ groups might be less known, we have collected some relevant facts in Appendix A. The spectra for the

pure line bundle models agree with those given in Ref. [24]; the other spectra are novel except that of the standard embedding.

\subsection{Supersymmetric blowups}

We study blow-ups of the $\mathbb{Z}_{3}$ heterotic orbifold models, that preserve six dimensional supersymmetry by switching on VEV's for twisted and possibly also untwisted states, and that can be identified with the smooth bundle models listed in Table 3. The analysis can be performed entirely at the classical level, because in six dimensional super-Yang-Mills theory dangerous loop corrections to the potential are absent. (This is of course unlike the four dimensional case, where one-loop Fayet-Iliopoulos corrections may arise.)

The study of flat directions of the potential $V$ involves the three real auxiliary fields, $D_{a}^{i}$ with $i=1,2,3$, of six dimensional super Yang-Mills theory

$$
V=\frac{1}{2} \sum_{i, a}\left(D_{a}^{i}\right)^{2}, \quad D_{a}^{i}=\sigma_{\alpha \beta}^{i} \phi_{\alpha}^{\dagger} T_{a} \phi_{\beta},
$$

where the representation indices on the complex scalar components $\phi_{1}, \phi_{2}$ of a hypermultiplet and gauge generator $T_{a}$ have been suppressed. It turns out convenient to use four dimensional $\mathcal{N}=1$ notation of a real D-term $D_{a}=D_{a}^{3}$ and a complex F-term $F_{a}=\left(D_{a}^{1}+i D_{a}^{2}\right) / \sqrt{2}$. Since the complex scalar $\phi_{1}$ and $\phi_{2}$ components of hypermultiplets are in complex conjugate representations, we have

$$
V=\frac{1}{2} \sum_{a} D_{a}^{2}+\sum_{a} \bar{F}_{a} F_{a}, \quad D_{a}=\bar{\phi}_{1} T_{a} \phi_{1}-\phi_{2} T_{a} \bar{\phi}_{2}, \quad \bar{F}_{a}=\phi_{2} T_{a} \phi_{1} .
$$




\begin{tabular}{|c|c|c|c|}
\hline$\left(n_{1}^{1}, n_{3}^{\frac{1}{2}}, n_{2}^{\frac{1}{2}}, n_{1}^{\frac{1}{2}}\right)$ & $V_{1}=-V_{2}$ & Non-Abelian gauge group & Matter spectrum (up to singlets) \\
\hline$(2,0,0,0)$ & $\left(0^{16}\right)$ & $\mathrm{SO}(26)$ & $2(\mathbf{2 6})$ \\
\hline$(1,0,0,2)$ & $\left(0^{16}\right)$ & $\mathrm{SO}(21) \times \mathrm{Sp}(4)$ & $\frac{1}{9}(\mathbf{2 1}, \mathbf{4})+(\mathbf{2 1}, \mathbf{1})+(\mathbf{1}, \mathbf{5})+3(\mathbf{1}, \mathbf{4})$ \\
\hline$(0,1,0,0)$ & $\left(0^{16}\right)$ & $\mathrm{SO}(28) \times \mathrm{SU}(2)$ & $\frac{10}{9}(\mathbf{2 8}, \mathbf{2})$ \\
\hline$(0,0,0,4)$ & $\left(0^{16}\right)$ & $\mathrm{SO}(16) \times \mathrm{Sp}(8)$ & $\frac{1}{9}(\mathbf{1 6}, \mathbf{8})+(\mathbf{1}, \mathbf{2 7})$ \\
\hline$(1,0,0,1)$ & $\begin{array}{c}(\overbrace{1,-1}, 0^{14}) \\
\left(1^{2}, 0^{14}\right)\end{array}$ & $\begin{array}{l}\mathrm{SO}(25) \\
\mathrm{SO}(21) \times \mathrm{SU}(2)^{2}\end{array}$ & $\begin{array}{l}\frac{17}{9}(\mathbf{2 5}) \\
\frac{1}{9}(\mathbf{2 1}, \mathbf{2}, \mathbf{1})+\frac{1}{9}(\mathbf{2 1}, \mathbf{1}, \mathbf{2})+(\mathbf{2 1}, \mathbf{1}, \mathbf{1})+\frac{8}{9}(\mathbf{1}, \mathbf{2}, \mathbf{2}) \\
+3(\mathbf{1}, \mathbf{2}, \mathbf{1})+3(\mathbf{1}, \mathbf{1}, \mathbf{2})\end{array}$ \\
\hline$(1,0,0,0)$ & $\begin{array}{l}\left(2^{2}, 0^{14}\right) \\
\left(1^{4}, 0^{12}\right)\end{array}$ & $\begin{array}{l}\mathrm{SO}(27) \\
\mathrm{SO}(21) \times \mathrm{SU}(4)\end{array}$ & $\begin{array}{l}\frac{19}{9}(\mathbf{2 7}) \\
\frac{1}{9}(\mathbf{2 1}, \mathbf{4})+\frac{10}{9}(\mathbf{1}, \mathbf{6})+3(\mathbf{1}, \mathbf{4})+(\mathbf{2 1}, \mathbf{1})\end{array}$ \\
\hline$(0,0,0,3)$ & $\begin{array}{c}(\overbrace{1,-1}, 0^{14}) \\
\left(1^{2}, 0^{14}\right)\end{array}$ & $\begin{array}{l}\mathrm{SO}(20) \times \mathrm{Sp}(4) \\
\mathrm{SO}(16) \times \mathrm{Sp}(6) \times \mathrm{SU}(2)\end{array}$ & $\begin{array}{l}\frac{1}{9}(\mathbf{2 0}, \mathbf{4})+\frac{8}{9}(\mathbf{2 0}, \mathbf{1})+\frac{28}{9}(\mathbf{1}, \mathbf{4})+(\mathbf{1}, \mathbf{5}) \\
\frac{1}{9}(\mathbf{1 6}, \mathbf{1}, \mathbf{2})+\frac{1}{9}(\mathbf{1 6}, \mathbf{6}, \mathbf{1})+\frac{8}{9}(\mathbf{1}, \mathbf{6}, \mathbf{2})+(\mathbf{1}, \mathbf{1 4}, \mathbf{1})\end{array}$ \\
\hline$(0,0,0,2)$ & $\begin{array}{l}\overbrace{(\overbrace{1,-1}^{2},-1^{2}}^{\left(2,0^{15}\right)}, 0^{2}) \\
\overbrace{\frac{1}{2}(\overbrace{1^{2},-1^{2}}{ }^{12}}, 1^{12})\end{array}$ & $\begin{array}{l}\mathrm{SO}(22) \times \mathrm{Sp}(4) \\
\mathrm{SO}(24) \\
\mathrm{SO}(20) \times \mathrm{SU}(2)^{2} \\
\mathrm{SO}(16) \times \mathrm{Sp}(4) \times \mathrm{SU}(4) \\
\mathrm{SU}(12)\end{array}$ & $\begin{array}{l}\frac{1}{9}(\mathbf{2 2}, \mathbf{4})+\frac{10}{9}(\mathbf{2 2}, \mathbf{1})+(\mathbf{1}, \mathbf{5})+\frac{26}{9}(\mathbf{1}, \mathbf{4}) \\
\frac{16}{9}(\mathbf{2 4}) \\
\frac{1}{9}(\mathbf{2 0}, \mathbf{2}, \mathbf{1})+\frac{1}{9}(\mathbf{2 0}, \mathbf{1}, \mathbf{2})+\frac{8}{9}(\mathbf{2 0}, \mathbf{1}, \mathbf{1})+\frac{8}{9}(\mathbf{1}, \mathbf{2}, \mathbf{2}) \\
+\frac{28}{9}(\mathbf{1}, \mathbf{1}, \mathbf{2})+\frac{28}{9}(\mathbf{1}, \mathbf{2}, \mathbf{1}) \\
\frac{1}{9}(\mathbf{1 6}, \mathbf{1}, \mathbf{4})+\frac{1}{9}(\mathbf{1 6}, \mathbf{4}, \mathbf{1})+\frac{10}{9}(\mathbf{1}, \mathbf{1}, \mathbf{6})+\frac{8}{9}(\mathbf{1}, \mathbf{4}, \mathbf{4}) \\
+(\mathbf{1}, \mathbf{5}, \mathbf{1}) \\
\frac{20}{9}(\mathbf{1 2})+\frac{1}{9}(\mathbf{6 6})\end{array}$ \\
\hline$(0,0,0,1)$ & $\begin{array}{l}(2, \overbrace{1,-1}^{\left(2,1^{2}, 0^{13}\right)}) \\
(\overbrace{1,-1}^{13}, 1^{4}, 0^{10}) \\
(\overbrace{}^{6}, 0^{10}) \\
(\overbrace{1,-1}^{1,}, 1^{13}, 3)\end{array}$ & $\begin{array}{l}\mathrm{SO}(26) \\
\mathrm{SO}(22) \times \mathrm{SU}(2)^{2} \\
\mathrm{SO}(20) \times \mathrm{U}(4) \\
\mathrm{SO}(16) \times \mathrm{SU}(6) \times \mathrm{SU}(2) \\
\mathrm{SU}(13)\end{array}$ & $\begin{array}{l}2(\mathbf{2 6}) \\
\frac{1}{9}(\mathbf{2 2}, \mathbf{2}, \mathbf{1})+\frac{1}{9}(\mathbf{2 2}, \mathbf{1}, \mathbf{2})+\frac{10}{9}(\mathbf{2 2}, \mathbf{1}, \mathbf{1})+\frac{8}{9}(\mathbf{1}, \mathbf{2}, \mathbf{2}) \\
+\frac{26}{9}(\mathbf{1}, \mathbf{2}, \mathbf{1})+\frac{26}{9}(\mathbf{1}, \mathbf{1}, \mathbf{2}) \\
\frac{1}{9}(\mathbf{2 0}, \mathbf{4})+\frac{8}{9}(\mathbf{2 0}, \mathbf{1})+\frac{28}{9}(\mathbf{1}, \mathbf{4})+\frac{10}{9}(\mathbf{1}, \mathbf{6}) \\
\frac{1}{9}(\mathbf{1 6 , 6}, \mathbf{1})+\frac{1}{9}(\mathbf{1 6}, \mathbf{1}, \mathbf{2})+\frac{8}{9}(\mathbf{1}, \mathbf{6}, \mathbf{2})+\frac{10}{9}(\mathbf{1}, \mathbf{1 5}, \mathbf{1}) \\
\frac{21}{9}(\mathbf{1 3})+\frac{1}{9}(\mathbf{7 8})\end{array}$ \\
\hline$(0,0,0,0)$ & $\begin{array}{l}\left(2^{2}, 0^{14}\right) \\
\left(2,1^{4}, 0^{11}\right) \\
\left(1^{8}, 0^{8}\right) \\
\frac{1}{2}\left(1^{14}, 3^{2}\right)\end{array}$ & $\begin{array}{l}\mathrm{SO}(28) \times \mathrm{SU}(2) \\
\mathrm{SO}(22) \times \mathrm{SU}(4) \\
\mathrm{SO}(16) \times \mathrm{SU}(8) \\
\mathrm{SU}(14) \times \mathrm{SU}(2)\end{array}$ & $\begin{array}{l}\frac{10}{9}(\mathbf{2 8}, \mathbf{2}) \\
\frac{1}{9}(\mathbf{2 2}, \mathbf{4})+\frac{10}{9}(\mathbf{2 2}, \mathbf{1})+\frac{10}{9}(\mathbf{1}, \mathbf{6})+\frac{26}{9}(\mathbf{1}, \mathbf{4}) \\
\frac{1}{9}(\mathbf{1 6}, \mathbf{8})+\frac{10}{9}(\mathbf{1}, \mathbf{2 8}) \\
\frac{1}{9}(\mathbf{9 1}, \mathbf{1})+\frac{11}{9}(\mathbf{1 4}, \mathbf{2})\end{array}$ \\
\hline
\end{tabular}

Table 3: This table gives the Abelian and non-Abelian bundles fulfilling the local Bianchi identity (66) on the resolved $\mathbb{C}^{2} / \mathbb{Z}_{3}$ singularity, and the resulting models. The first column indicates the instanton numbers of the non-Abelian bundles. The second column gives the Abelian bundle vectors. The overbrace indicates the amount of "overlap", i.e. shared Cartan generators, there is between Abelian background and the $\mathrm{SU}(2)$ instanton(s). The third column lists the possible unbroken non-Abelian gauge group. The final column gives the resulting spectrum up to singlets. 


\begin{tabular}{|c|c|c|c|c|c|}
\hline$\left(n_{1}^{1}, n_{3}^{\frac{1}{2}}, n_{2}^{\frac{1}{2}}, n_{1}^{\frac{1}{2}}\right)$ & $V_{1}=-V_{2}$ & Unbroken gauge group & $\#$ & Twisted & Untwisted \\
\hline$(2,0,0,0)$ & $\left(0^{16}\right)$ & $\mathrm{SO}(26)$ & $3 \mathrm{a}$ & $(28,2),(1)$ & \\
\hline$(1,0,0,2)$ & $\left(0^{16}\right)$ & $\mathrm{SO}(21) \times \mathrm{Sp}(4)$ & $3 b$ & $(22,1),(1,10)$ & $(22,5)$ \\
\hline$(0,1,0,0)$ & $\left(0^{16}\right)$ & $\mathrm{SO}(28) \times \mathrm{SU}(2)$ & $3 a$ & $2 \times(\mathbf{1})$ & \\
\hline$(0,0,0,4)$ & $\left(0^{16}\right)$ & $\mathrm{SO}(16) \times \mathrm{Sp}(8)$ & $3 \mathrm{c}$ & $\begin{array}{l}(1,28),(1) \\
(1,28),(1)\end{array}$ & $(1,28)$ \\
\hline$(1,0,0,1)$ & $\begin{array}{l}(\overbrace{1,-1}, 0^{14}) \\
\left(1^{2}, 0^{14}\right)\end{array}$ & $\begin{array}{l}\mathrm{SO}(25) \\
\mathrm{SO}(21) \times \mathrm{SU}(2) \times \mathrm{SU}(2)\end{array}$ & $\begin{array}{l}3 \mathrm{a} \\
3 \mathrm{~b}\end{array}$ & $\begin{array}{l}(28,2),(1) \\
(22,1),(1,10),(1,5)\end{array}$ & $\begin{array}{l}(28,2) \\
(1,10)\end{array}$ \\
\hline$(1,0,0,0)$ & $\begin{array}{l}\left(2,0^{15}\right) \\
\left(1^{4}, 0^{12}\right)\end{array}$ & $\begin{array}{l}\mathrm{SO}(27) \\
\mathrm{SO}(21) \times \mathrm{SU}(4)\end{array}$ & $\begin{array}{l}3 \mathrm{a} \\
3 \mathrm{~b}\end{array}$ & $\begin{array}{l}(28,2),(1) \\
(22,1),(1,5)\end{array}$ & $\begin{array}{l}(28,2) \\
(22,5)\end{array}$ \\
\hline$(0,0,0,3)$ & $\begin{array}{l}(\overbrace{1,-1}^{1}, 0^{14}) \\
\left(1^{2}, 0^{14}\right)\end{array}$ & $\begin{array}{l}\mathrm{SO}(20) \times \mathrm{Sp}(4) \\
\mathrm{SO}(16) \times \mathrm{Sp}(6) \times \mathrm{SU}(2)\end{array}$ & $\begin{array}{l}3 \mathrm{~b} \\
3 \mathrm{c}\end{array}$ & $\begin{array}{l}(22,1),(1,10) \\
(1,28),(1)\end{array}$ & $\begin{array}{l}(22,5) \\
(1,28)\end{array}$ \\
\hline$(0,0,0,2)$ & 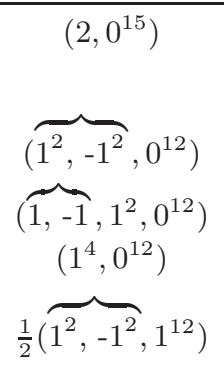 & $\begin{array}{l}\mathrm{SO}(22) \times \mathrm{Sp}(4) \\
\mathrm{SO}(24) \\
\mathrm{SO}(20) \times \mathrm{SU}(2)^{2} \\
\mathrm{SO}(16) \times \mathrm{Sp}(4) \times \mathrm{SU}(4) \\
\mathrm{SU}(12)\end{array}$ & $\begin{array}{l}3 \mathrm{~b} \\
3 \mathrm{a} \\
3 \mathrm{~b} \\
3 \mathrm{c} \\
3 \mathrm{e}\end{array}$ & $\begin{array}{l}(1,10),(1,5) \\
(1,10),(1,5) \\
(28,2),(1) \\
(22,1),(1,10) \\
(1,28),(1) \\
(14,2,1),(2,1,1),(1)\end{array}$ & $\begin{array}{l}(1,10) \\
(28,2) \\
(22,5),(1,10) \\
(1,28) \\
(14,2,2)\end{array}$ \\
\hline$(0,0,0,1)$ & 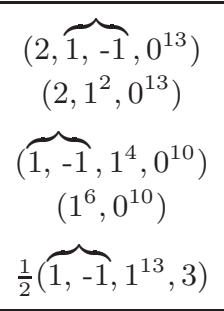 & $\begin{array}{l}\mathrm{SO}(26) \\
\mathrm{SO}(22) \times \mathrm{SU}(2)^{2} \\
\mathrm{SO}(20) \times \mathrm{U}(4) \\
\mathrm{SO}(16) \times \mathrm{SU}(2) \times \mathrm{SU}(6) \\
\mathrm{SU}(13)\end{array}$ & $\begin{array}{l}3 \mathrm{a} \\
3 \mathrm{~b} \\
3 \mathrm{~b} \\
3 \mathrm{c} \\
3 \mathrm{e}\end{array}$ & $\begin{array}{l}(28,2),(1) \\
(1,10),(1,5) \\
(22,1),(1,5) \\
(1,28),(1) \\
(14,2,1),(2,1,1),(1)\end{array}$ & $\begin{array}{l}(1,10) \\
(22,5) \\
(1,28) \\
(14,2,2)\end{array}$ \\
\hline$(0,0,0,0)$ & $\begin{array}{c}\left(2^{2}, 0^{14}\right) \\
\left(2,1^{4}, 0^{11}\right) \\
\left(1^{8}, 0^{8}\right) \\
\frac{1}{2}\left(1^{14}, 3^{2}\right)\end{array}$ & $\begin{array}{l}\mathrm{SO}(28) \times \mathrm{SU}(2) \\
\mathrm{SO}(22) \times \mathrm{SU}(4) \\
\mathrm{SO}(16) \times \mathrm{SU}(8) \\
\mathrm{SU}(14) \times \mathrm{SU}(2)\end{array}$ & $\begin{array}{l}3 \mathrm{a} \\
3 \mathrm{~b} \\
3 \mathrm{c} \\
3 \mathrm{e}\end{array}$ & $\begin{array}{l}2 \times(\mathbf{1}) \\
2 \times(\mathbf{1}, \mathbf{5}) \\
2 \times(\mathbf{1}) \\
2 \times(\mathbf{1}, \mathbf{1}, \mathbf{2})\end{array}$ & \\
\hline
\end{tabular}

Table 4: The first three columns contain the same information as Table 3. The final columns indicate from which of five heterotic $\mathbb{Z}_{3}$ models, listed in Table 1 , these bundle models can be obtained by switching on VEV's for the indicated twisted and untwisted states. 
Therefore, if the scalars in the hypermultiplet are internally aligned, i.e.

$$
\phi_{2}=\alpha_{\phi} \bar{\phi}_{1}, \quad\left|\alpha_{\phi}\right|=1 \Rightarrow \bar{F}=\alpha_{\phi} \bar{\phi}_{1} T_{a} \phi_{1},
$$

the $\mathrm{D}$-term vanishes immediately, and the $\mathrm{F}$-term takes the form of a $\mathrm{D}$-term but with a phase $\alpha_{\phi}$ as pre-factor. If one has more than one hypermultiplet, the alignment can happen in each hyper multiplet separately, which gives a collection of phases, and relative signs in particular. This simplifies the analysis considerably: One does not have to worry anymore about D-terms and the phases may be used to make the $\mathrm{F}$-terms vanish as well.

The subsequent analysis of the flat directions is straightforward but somewhat tedious. We have diverted most of this discussion to Appendix C; here we only summarize the results of the complete analysis in Table 4. In this table we list for each of the bundle models given in Table 3 from which heterotic orbifold models, classified in Table 1, it can be obtained by switching on the VEV's for the hypermultiplets listed in the last column of Table 4. This table shows that each bundle model, for which explicit solutions to the Hermitean Yang-Mills equations exist, indeed corresponds to an Fand D-flat direction.

One can follow this correspondence of the bundle models and the orbifold models also at the level of the spectra. We have checked, that the non-Abelian spectra of the orbifold models, with appropriate VEV's switched on, results in branching of the matter representation giving precisely the non-Abelian spectra of the bundle models. This identification is exact if one takes Higgsing of vector multiplets due to symmetry breaking into account, that eats away some hypermultiplets. Because of six dimensional chirality, states can only pair up and become massive, provided that one is a vector multiplet and the other a hypermultiplet. This means that the index theorem exactly determines the number of massless hyper (including non-Abelian singlets) and vector multiplets. In Table 3 we refrained from giving the multiplicities of singlet states; they can either be directly computed via the index theorem, or using the fact that the pure gravitational anomaly gives a relation between the number of vector multiplets and hypermultiplets [61,62].

From Table 4 we can determine some relations between the VEV's of twisted and untwisted states of the orbifold model and the corresponding bundle model. In particular, we see that all pure line bundle models are obtained by switching on VEV's for two identical twisted hypers. All other bundle models have different hypermultiplets switched on; except for the standard embedding model with $n=(0,1,0,0)$.

\subsection{Modified local Bianchi identity}

The comparison between possible VEV configurations of orbifold models and the explicit bundle models constructed here, indicates that our list of bundles is not complete: There are supersymmetric VEV assignments that do not seem to have a counter part as a bundle model. Before we explain what is going on here, we first give two examples of this situation:

First of all, notice that all the constructed bundle models are obtained by switching on VEV's in orbifold models 3a, 3b, 3c and 3e of Table 1, while model 3d is never used. Nevertheless this model has a fully flat direction with simultaneously suitably aligned VEV's of the $(\mathbf{1 0}, \mathbf{1 1})_{1},(\mathbf{1}, \mathbf{5 5})_{2}$ and $(\mathbf{1}, \mathbf{1 1})_{-8 / 3}$ breaking the gauge group to $\mathrm{SO}(9) \times \mathrm{Sp}(10)$.

A second example is provided by orbifold model 3c. We see from Table 4 that all the bundle models with an $\mathrm{SO}(16)$ group factor result from this orbifold model by switching on VEV's of one or two twisted singlets $(\mathbf{1})_{8 / 3}$ and the twisted and untwisted anti-symmetric tensors, $(\mathbf{1}, \mathbf{2 8})_{-2 / 3}$ and $(\mathbf{1}, \mathbf{2 8})_{1}$. 
Following the analysis of Appendix $\mathrm{C}$ one concludes that with these multiplets taking VEV's the possible unbroken gauge group could be any of the ones listed in Table 5 . These different possibilities arise because of the VEV's for the anti-symmetric tensors: They can be skew-diagonalized. Then depending on whether some or all of its diagonal entries are equal and / or zero, one of the above mentioned gauge groups is realized. (For example: All entries zero gives SU(8), all entries equal but non-zero gives $\mathrm{Sp}(8)$, and finally all entries different gives $\mathrm{SU}(2)^{4}$.) Table 3 does not contain the gauge group factors $\mathrm{Sp}(4) \times \mathrm{Sp}(4), \mathrm{SU}(4) \times \mathrm{SU}(2)^{2}, \mathrm{Sp}(4) \times \mathrm{SU}(2)^{2}$ and $\mathrm{SU}(2)^{4}$, hence there are bundle models missing.

As a side remark we note, that this example also shows that many different bundle models, characterized by different topological parameters are actually related to each other by continuous deformations of the VEV's of twisted and untwisted states of the corresponding orbifold model. The precise relation between the moduli space of VEV configurations and bundle models is beyond the scope of this paper. Presumably this requires to analyze the full gauge bundle moduli space using the ADHM [44,45] and Kronheimer-Nakajima [46-48] constructions, see e.g. [36,37, 49,63].

Bundle realizations of these and other VEV configurations of orbifold models can be obtained realizing that the local Bianchi identity (66) is a sufficient condition to uncover consistent models but certainly not a necessary condition. Indeed, only on a compact K3 the integrated Bianchi identity needs to vanish. This means that if one has a compact orbifold, say like $T^{4} / \mathbb{Z}_{3}$, the sum of the instanton numbers from all fixed points needs to equal 24. The local Bianchi identity (66) is obtained by splitting up the total instanton number of K3 equally over all 9 fixed points of $T^{4} / \mathbb{Z}_{3}$. The total instanton number 24 cannot be completely arbitrarily distributed over the various fixed points, since the instanton number is quantized itself $[55,64,65]$ : The basic unit of instanton number that can be moved around equals 1 . This means that the local Bianchi identity (66) equals $K=8 / 3 \bmod 1$. The additional constraint, that the first Chern class of the bundle is even, implies that $K=8 / 3 \bmod 2$,

unless $n_{2}^{1 / 2}$ is odd. This coincides precisely with the (weak) modular invariance condition for a local orbifold shift vector.

The smallest local Bianchi identity has $K=2 / 3$ in (66). There are two solutions to this equation: i) $n_{1}^{1 / 2}=1, V_{1}=V_{2}=0$, which results in the unbroken gauge group $\mathrm{SO}(28) \times \mathrm{SU}(2)$, and ii) $n_{1}^{1 / 2}=0$, $V_{1}=-V_{2}=\left(1^{2}, 0^{14}\right)$, with unbroken gauge group $\mathrm{SO}(28) \times \mathrm{SU}(2)$. Thus both are VEV configurations of orbifold model $3 \mathrm{a}$, which we had already found.

Using the modified Bianchi identity, eq. (66) for an instanton number $K=14 / 3$ a bundle realization of the VEV configuration of model $3 \mathrm{~d}$ can be found: The bundle is characterized by $n_{1}^{1}=1$ and $n_{1}^{1 / 2}=$ 5. Also the VEV configurations of $3 c$ with gauge groups $\mathrm{Sp}(4) \times \mathrm{Sp}(4), \mathrm{SU}(4) \times \mathrm{SU}(2)^{2}, \mathrm{Sp}(4) \times \mathrm{SU}(2)^{2}$ and $\mathrm{SU}(2)^{4}$, discussed above, can be identified. For each of these models we give a bundle candidate in Table 5. To compute the spectra of these models is challenging because for that we need a modified index theorem that takes the non-vanishing three-form flux $H_{3}$ into account. Indeed, using the standard index theorem ensures an anomaly-free spectrum only in case the Bianchi identity is fulfilled $[61,66]$.

\section{Conclusions and outlook}

The construction of stable non-Abelian bundles on Calabi-Yau manifolds is one of the outstanding problems in both mathematics and theoretical physics. Yet to determine the full phenomenological potential of heterotic string constructions this is of fundamental importance. In this paper we exploited 


\begin{tabular}{|c|c|c|}
\hline gauge group & bundle realization & $K$ \\
\hline \hline $\mathrm{SO}(16) \times \mathrm{SU}(8)$ & $V_{1}=\left(1^{8}, 0^{8}\right)$ & $\frac{8}{3}$ \\
$\mathrm{SO}(16) \times \mathrm{Sp}(8)$ & $n_{1}^{1 / 2}=4$ & $\frac{8}{3}$ \\
$\mathrm{SO}(16) \times \mathrm{Sp}(6) \times \mathrm{SU}(2)$ & $n_{1}^{1 / 2}=3, V_{1}=\left(1^{2}, 0^{14}\right)$ & $\frac{8}{3}$ \\
$\mathrm{SO}(16) \times \mathrm{SU}(4) \times \mathrm{Sp}(4)$ & $n_{1}^{1 / 2}=2, V_{1}=\left(1^{4}, 0^{12}\right)$ & $\frac{8}{3}$ \\
\hline \hline $\mathrm{SO}(16) \times \mathrm{SU}(6) \times \mathrm{SU}(2)$ & $n_{1}^{1 / 2}=1, V_{1}=\left(1^{6}, 0^{8}\right)$ & $\frac{8}{3}$ \\
$\mathrm{SO}(16) \times \mathrm{Sp}(4) \times \mathrm{Sp}(4)$ & $n_{2}^{1 / 2}=n_{1}^{1 / 2}=2$ & $\frac{14}{3}$ \\
$\mathrm{SO}(16) \times \mathrm{SU}(4) \times \mathrm{SU}(2)^{2}$ & $n_{1}^{1 / 2}=1, V_{1}=\left(2^{2}, 1^{4}, 0^{10}\right)$ & $\frac{14}{3}$ \\
$\mathrm{SO}(16) \times \mathrm{Sp}(4) \times \mathrm{SU}(2)^{2}$ & $n_{1}^{1 / 2}=2, V_{1}=\left(2^{2}, 1^{2}, 0^{12}\right)$ & $\frac{14}{3}$ \\
\hline \hline $\mathrm{SO}(16) \times \mathrm{SU}(2)^{4}$ & $n_{3}^{1 / 2}=n_{1}^{1 / 2}=1, V_{1}=\left(2^{2}, 1^{2}, 0^{12}\right)$ & $\frac{20}{3}$ \\
\hline
\end{tabular}

Table 5: We give possible bundle realizations of all VEV configurations of model 3c. The last column indicates for which models a modification of the local Bianchi identity is required, i.e. $K \neq 8 / 3$. (Only the non-vanishing gauge instanton numbers are given, and $V_{1}=-V_{2}$ is assumed.)

the fact that well-known instantons on Eguchi-Hanson spaces provide explicit examples of stable bundles on non-compact four dimensional $\mathbb{C}^{2} / \mathbb{Z}_{n}$ orbifold blowups with non-Abelian structure groups. Because in addition also line bundles have been constructed on Eguchi-Hanson spaces explicitly, we have access to a substantial class of bundles that can be used for six dimensional model building. Using this we gave a complete classification of all possible combinations of these instantons with Abelian gauge fluxes, that fulfill the local Bianchi identity constraint on the $\mathbb{C}^{2} / \mathbb{Z}_{3}$ resolution. Spectra were computed using index theorems; to obtain anomaly-free spectra it was crucial that the Bianchi identity was fulfilled locally. The resulting effective six dimensional models have been listed in Table 3 .

All of these gauge backgrounds can be related to a configurations of VEV's of states present in the corresponding heterotic orbifold models. For models with only Abelian gauge fluxes always two identical twisted hypermultiplets take VEV's, confirming our previous findings [24,25,32], that line bundle models correspond to orbifold models with a single twisted VEV switched on. For non-Abelian gauge fluxes or gauge backgrounds that combine both line bundles and bundles with non-Abelian structure groups, we always need combinations of simultaneous VEV's of twisted and often even untwisted states to identify matching orbifold constructions. In all cases we confirmed that both the gauge groups and spectra are identical in the orbifold and bundle perspectives. The multiplicities of states in the smooth construction, computed using the index theorem on local resolutions, seems to take rather arbitrary values, given in the final column of Table 3. All these values can be understood from the orbifold perspective as the combination of twisted states with integral multiplicities, untwisted states with multiplicity 1/9 (because they are bulk modes shared between nine orbifold fixed points), and the effect of Higgsings that take away multiples of $1 / 9$. Therefore, this provides stringent consistency checks on our results. 
We have shown that each combination of instantons and Abelian gauge fluxes that fulfill the local Bianchi identity corresponds to a VEV configuration of a certain heterotic orbifold. One may wonder whether one can reverse the statement: Each supersymmetric system of VEV's correspond to a configuration of instantons and gauge fluxes. Presumably this statement is true, but certainly not all these configurations satisfy the local Bianchi identities. Indeed, we observed that model $3 \mathrm{~d}$ of Table 1 is not used at all as an orbifold realization of a bundle model that satisfies this condition, see Table 4, even though it definitely possesses flat directions. If we give up the local Bianchi identity and allow that it differs by some instanton units, a configuration can be identified that leads to the same gauge group as one obtains from the VEV configuration. To confirm the matching on the level of the spectra is hampered by the fact, that index theorems on non-compact spaces cannot be employed when the local Bianchi is not satisfied. A generalization of the index theorem in the presence of the corresponding three form $H$-flux is needed.

The situation is similar for the possible VEV configurations of the other orbifold models. For concreteness we focused on model 3c: Only some of its VEV configurations are realized as bundle models satisfying the local Bianchi identity. Other VEV assignments can only be realized, when it is only fulfilled up to a number of instanton units. The resulting Bianchi identity is then very similar to the modular invariance condition of heterotic orbifolds. All these different bundle models correspond to VEV configurations which are all continuously connected to each other. Different bundle models often only correspond to very similar VEV configurations, except that in one case the VEVs are equal, in the other they are different. One does not need to take large numbers of VEVs to zero to interpolate between such configurations, therefore these transitions are deformations of the bundle rather than flops. In light of this one may wonder what the topological classification of the bundles exactly means. The description of bundles on Eguchi-Hanson spaces employed by us is not the most general: The Kronheimer-Nakajima construction [47] describes the full moduli space on such ALE gravitational instantons, and might therefore be a more appropriate setting for this comparison.

Most of the findings reported in this work relied on the crucial fact that on Eguchi-Hanson spaces, Abelian gauge backgrounds and non-Abelian instanton configurations are known. Explicit resolutions of $\mathbb{C}^{3} / \mathbb{Z}_{n}$ for $n>3$ orbifolds are not known, hence to have access to bundles with non-Abelian structure groups on $\mathbb{C}^{3} / \mathbb{Z}_{n}$ resolutions is much more challenging. (Of course one always has the standard embedding, but precisely since it immediately fulfills the local Bianchi identity, it only corresponds to one configuration.) Yet this is of great importance because there are certain six dimensional orbifolds, like the $T^{6} / \mathbb{Z}_{6 \text {-II }}$ for which a large pool of MSSM-like models have been constructed recently. Resolutions of generic $\mathbb{C}^{3} / \mathbb{Z}_{n}$ orbifolds and their line bundles are only known in toric geometry. In the hope to find a framework that allows us to describe stable bundles with non-Abelian structure groups on toric resolutions of such orbifold singularities, we reformulated the description of the Eguchi-Hanson instantons in terms of a toric geometry-like language.

\section{Acknowledgments}

We would like to thank Massimo Bianchi, Kang-Sin Choi, Tae-Won Ha, Arthur Hebecker, Maximilian Kreuzer, Michael Ratz, Emanuel Scheidegger, Stefan Vandoren and Jenny Wagner for stimulating discussions and correspondence. F.P.C. is grateful to the Institut fur Theoretische Physik, Heidelberg, Germany, for support and hospitality during visits in the early stage of this work. The work of F.P.C is supported by FCT through the grant SFRH/BPD/20667/2004. The work of MT is supported by the European Community through the contract N 041273 (Marie Curie Intra-European Fellowships). 
He is also partially supported by the ANR grant ANR-05-BLAN-0079-02, the RTN contracts MRTNCT-2004-005104 and MRTN-CT-2004-503369, the CNRS PICS \# 2530, 3059 and 3747, and by the European Union Excellence Grant MEXT-CT-2003-509661.

\section{A Some $\operatorname{Sp}(2 \mathrm{n})$ representation theory}

This Appendix is devoted to some elementary properties of representation of $\operatorname{Sp}(2 n)$ groups and how they arise in branching from $\mathrm{SO}(4 n)$ groups. $\mathrm{Sp}(2 n)$ groups are less common in physics, for that reason we review the properties that we need here. (See for a more extensive discussion Ref. [67].) The group $\operatorname{Sp}(2 n)$ is defined as the group of real matrices that leave a symplectic form (anti-symmetric $2 n \times 2 n$ matrix) $\Omega$ invariant

$$
S^{T} \Omega S=\Omega, \quad \Omega=\mathbb{1}_{n} \otimes \epsilon=\left(\begin{array}{cc}
0 & \mathbb{1}_{n} \\
-\mathbb{1}_{n} & 0
\end{array}\right)
$$

The form of the symplectic matrix $\Omega$ given here can be obtained by a suitable basis choice. Alternatively one can define this group as the set of unitary matrices $U \in \mathrm{SU}(2 \mathrm{n})$ that leave this symplectic form invariant $U^{\dagger} \Omega U=\Omega$. This group is then also often referred to as $\operatorname{USp}(2 n)$, both definitions in fact define the same abstract group.

We list the basic representations of $\operatorname{Sp}(2 n)$. Since $\operatorname{Sp}(2 n)$ is defined as a matrix group, its fundamental representation is the $2 n$ component vector representation $\mathbf{2 n}$ on which these matrices act naturally. The adjoint representation is defined as the algebra of the group. Writing an algebra element $A$ as a block matrix, we find that its matrix blocks satisfy

$$
A=\left(\begin{array}{ll}
\alpha & \beta \\
\gamma & \delta
\end{array}\right), \quad \beta^{T}=\beta, \quad \gamma^{T}=\gamma, \quad \delta=-\alpha^{T} .
$$

Therefore the adjoint consists of $n(2 n+1)$ components in total. This corresponds to symmetric Hermitian $2 n \times 2 n$ matrices, that are the generators of $\operatorname{Sp}(2 n)$ as a subgroup of the unitary group. We can also consider the anti-symmetric Hermitian matrices. This does not give directly an irreducible representation because the symplectic form $\Omega$ itself is anti-symmetric. Using it we can define the traceless anti-symmetric representation $[\mathbf{2 n}]_{2}$ with $n(2 n-1)-1$ components. These representations for $\operatorname{Sp}(2 n)$ groups up to $n=5$ are collected in Table 6 .

To compute the spectra of models when Sp-groups appear in the main part of the text the branching of $\mathrm{SO}(4 n)$ and $\mathrm{SU}(2 n)$ to $\mathrm{Sp}(2 n)$ are crucial. The relevant branching rules read

$$
\begin{array}{cl}
\mathrm{SO}(4 \mathrm{n}) & \rightarrow \mathrm{Sp}(2 \mathrm{n}) \times \mathrm{SU}(2) \\
\mathbf{4 n} & \rightarrow(\mathbf{2 n}, \mathbf{2}) \\
\mathbf{2 n}(\mathbf{4 n}-\mathbf{1}) & \rightarrow(\mathbf{n}(\mathbf{2 n}+\mathbf{1}), \mathbf{1})+(\mathbf{1}, \mathbf{3})+(\mathbf{n}(\mathbf{2 n}-\mathbf{1})-\mathbf{1}, \mathbf{3})
\end{array}
$$

and

$$
\begin{aligned}
\mathrm{SU}(2 \mathrm{n}) & \rightarrow \mathrm{Sp}(2 \mathrm{n}) \\
\mathbf{2 n} & \rightarrow \mathbf{2 n} \\
\mathbf{n}(\mathbf{2 n - 1}) & \rightarrow(\mathbf{n}(\mathbf{2 n - 1}) \mathbf{- 1})+(\mathbf{1}) \\
\mathbf{4} \mathbf{n}^{\mathbf{2}}-\mathbf{1} & \rightarrow(\mathbf{n}(\mathbf{2} \mathbf{n}+\mathbf{1}))+(\mathbf{n}(\mathbf{2 n - 1}) \mathbf{- 1})
\end{aligned}
$$




\begin{tabular}{|c||c|c|c|c|c|}
\hline$n$ & 1 & 2 & 3 & 4 & 5 \\
\hline $\operatorname{Sp}(2 n)$ & $\mathrm{Sp}(2)$ & $\mathrm{Sp}(4)$ & $\mathrm{Sp}(6)$ & $\mathrm{Sp}(8)$ & $\mathrm{Sp}(10)$ \\
\hline \hline $\mathbf{F u n d}=\mathbf{2 n}$ & $\mathbf{2}$ & $\mathbf{4}$ & $\mathbf{6}$ & $\mathbf{8}$ & $\mathbf{1 0}$ \\
\hline $\mathbf{A d}=\mathbf{n}(\mathbf{2 n}+\mathbf{1})$ & $\mathbf{3}$ & $\mathbf{1 0}$ & $\mathbf{2 1}$ & $\mathbf{3 6}$ & $\mathbf{5 5}$ \\
\hline$[\mathbf{2 n}]_{2}=\mathbf{n}(\mathbf{2 n - 1}) \mathbf{- 1}$ & - & $\mathbf{5}$ & $\mathbf{1 4}$ & $\mathbf{2 7}$ & $\mathbf{4 8}$ \\
\hline
\end{tabular}

Table 6: The elementary representations of the smallest $\operatorname{Sp}(2 n)$ groups are listed. The representations for the smallest two makes sense in view of the isomorphisms $\mathrm{Sp}(2)=\mathrm{SU}(2)$ and $\mathrm{Sp}(4)=\mathrm{SO}(5)$.

\section{B Clifford algebras for $\mathrm{SO}(4 \mathrm{~N})$}

In the main text we rely at certain points heavily on some properties of Clifford algebras and spinor representations of $\mathrm{SO}(\mathrm{N})$ groups. A convenient way of introducing their properties is to make use of an explicit basis. For the purposes of this paper we make the following choices. The standard Pauli matrices

$$
\sigma_{1}=\left(\begin{array}{ll}
0 & 1 \\
1 & 0
\end{array}\right), \quad \sigma_{2}=\left(\begin{array}{cc}
0 & -i \\
i & 0
\end{array}\right), \quad \sigma_{3}=\left(\begin{array}{cc}
1 & 0 \\
0 & -1
\end{array}\right),
$$

are defined such that $\sigma_{1} \sigma_{2}=i \sigma_{3}$.

The four dimensional Euclidean gamma matrices can be chosen as

$$
\gamma_{i}=\left(\begin{array}{cc}
0 & i \sigma_{i} \\
-i \sigma_{i} & 0
\end{array}\right), \quad \gamma_{4}=\left(\begin{array}{cc}
0 & \mathbb{1}_{2} \\
\mathbb{1}_{2} & 0
\end{array}\right)
$$

The spin generators $\frac{1}{2} \gamma_{A B}=\frac{1}{4}\left[\gamma_{A}, \gamma_{B}\right]$ of $\mathrm{SO}(4)$ are then given by

$$
\frac{1}{2} \gamma_{i j}=\frac{i}{2} \epsilon_{i j k}\left(\begin{array}{cc}
\sigma_{k} & 0 \\
0 & \sigma_{k}
\end{array}\right), \quad \frac{1}{2} \gamma_{k 4}=\frac{i}{2}\left(\begin{array}{cc}
\sigma_{k} & 0 \\
0 & -\sigma_{k}
\end{array}\right) .
$$

The product of all four gamma matrices defines the chirality operator $\gamma=\gamma_{1} \gamma_{2} \gamma_{3} \gamma_{4}$. Using it one defines the chiral projections of the spin generators

$$
\gamma_{A B}^{ \pm}=\gamma_{A B} P^{ \pm}, \quad P^{ \pm}=\frac{1 \pm \gamma}{2}
$$

Notice that $\gamma \gamma_{A B}=-\frac{1}{2} \epsilon_{A B C D} \gamma_{C D}$, hence positive chirality corresponds to self-duality, see (7) in our conventions.

The eight dimensional Euclidean Clifford algebra and spin group are obtained straightforwardly from the four dimensional one. We define

$$
\Gamma_{A}=\gamma_{A} \otimes \mathbb{1}, \quad \Gamma_{4+A}=\gamma \otimes \gamma_{A},
$$


as the basis of the generators of the Clifford algebra. The spin generators can be decomposed w.r.t. $\mathrm{SO}(4) \times \mathrm{SO}(4)$ as

$$
\Gamma_{A B}=\gamma_{A B} \otimes \mathbb{1}, \quad \Gamma_{4+A 4+B}=\mathbb{1} \otimes \gamma_{A B}, \quad \Gamma_{A 4+B}=\gamma_{A} \gamma \otimes \gamma_{B} .
$$

We denote the positive chirality spin generators for both $\mathrm{SO}(4)$ factors as $\Gamma_{A B}^{+}=\gamma_{A B}^{+} \otimes \mathbb{1}$ and $\Gamma_{4+A 4+B}^{+}=\mathbb{1} \otimes \gamma_{A B}^{+}$, respectively. The generators of spin $\mathrm{SO}(8)$ that commute with the sum $\Gamma_{A B}^{+}+$ $\Gamma_{4+A 4+B}^{+}$read

$$
\Gamma_{A B}^{-}=\gamma_{A B}^{-} \otimes \mathbb{1}, \quad \Gamma_{4+A 4+B}^{-}=\mathbb{1} \otimes \gamma_{A B}^{-}, \quad \Gamma_{A 4+A}=\gamma_{A} \gamma \otimes \gamma_{A}
$$

Together these elements generate $\operatorname{Sp}(4)$.

\section{Flatness analysis of $\mathbb{C}^{2} / \mathbb{Z}_{3}$ orbifold models}

Even though it is not the most general case, we assume internal alignment of the VEV's in hypermultiplets throughout the following analysis. Since our purpose is to find for each of the bundle models a realization as an heterotic orbifold theory with certain fields taking non-vanishing VEV's, this is sufficient for our purposes. This analysis has been divided into $\mathrm{U}(1), \mathrm{SU}(N), \mathrm{SO}(M)$ and product group flatness investigations below, as these are the gauge groups that appear in the model listed in Table 1.

\section{C.1 U(1) flatness}

To achieve U(1) flatness we need at least two hypermultiplets. If the hypermultiplets are charged under non-Abelian gauge multiplets one often needs more hypermultiplets to achieve the flatness for the other gauge symmetries as well. In particular, when one of the hyper multiplets, is a singlet w.r.t. to any non-Abelian gauge symmetry, the internal alignment phase and VEV can be adjusted to cancel the U(1) F-term. This means that if there are singlet hypermultiplets in the spectrum, U(1) flatness can always be achieved. Hence, from Table 1 we infer that in models $3 a, 3 c$ and $3 e \mathrm{U}(1)$ flatness can always be obtained, because they contain charged singlets. In all cases we enforce U(1) flatness only at the end because, it just gives a single extra condition which in most cases can be fulfilled easily by using singlets or by choosing relations between VEV's appropriately.

\section{C.2 SU(N) flatness}

The $\mathrm{F}$ - and $\mathrm{D}$-terms of an $\mathrm{SU}(N)$ gauge group can be represented as traceless $\mathrm{N} \times \mathrm{N}$ matrices. It is often more convenient to not enforce the tracelessness from the very beginning, but rather consider the $\mathrm{U}(N) \mathrm{F}$ - and D-terms represented by generic $N \times N$ matrices. Requiring that they are proportional to the identity, then enforces $\mathrm{SU}(N)$ flatness. In particular, after internal alignment has been used, $\mathrm{SU}(N)$ flatness requires that

$$
\bar{F}=\bar{f} \mathbb{1}_{N},
$$

where $\bar{f}$ is some complex number. For a single hypermultiplet $\phi=\left(\phi_{1}, \phi_{2}\right)$ in the fundamental representation, in which the $\phi_{1}$ is a $\mathrm{SU}(N)$ fundamental and the generators take the form $\left(T^{m n}\right)_{j k}=$ 
$\delta_{j}^{m} \delta_{k}^{n}$, the relation cannot be satisfied. Indeed, employing matrix notation we have

$$
\bar{F}=\alpha_{\phi} \phi_{1} \bar{\phi}_{1}
$$

This has determinant zero, and trace equal to $\bar{\phi}_{1} \phi_{1}$, but then the above requirement implies that $\phi_{1}$ vanishes identical. Notice that an additional charged singlet cannot help to fulfill the flatness condition.

\section{two fundamentals}

From these considerations we conclude that at least two hypermultiplets $\phi$ and $\psi$ in the fundamental representation are needed for $\mathrm{SU}(N)$ flatness. Assuming internal alignment the $\mathrm{F}$-term becomes

$$
\bar{F}=\alpha_{\phi} \phi_{1} \bar{\phi}_{1}+\alpha_{\psi} \psi_{1} \bar{\psi}_{1}
$$

In such a case cancellation can be ensured, by choosing $\alpha_{\phi}=-\alpha_{\psi}=1$ and $\psi_{1}=\phi_{1}$. Hence, we conclude that the fundamentals are aligned, and $\mathrm{SU}(N)$ is broken to $\mathrm{SU}(N-1)$. Notice that all the line bundle models are realized by either having two $\mathrm{SU}(N)$ vector or $\mathrm{U}(1)$ charged singlet representations a non-vanishing VEV, see the bottom part of Table 4.

\section{one antisymmetric tensor}

For a hypermultiplet $A=\left(A_{1}, A_{2}\right)$ in the antisymmetric representation of $\mathrm{SU}(N)$, i.e. $A^{m n}=-A^{n m}$, the flatness condition can be written as

$$
\bar{F}=\alpha_{A} A_{1} A_{1}^{\dagger}
$$

Using a $\mathrm{SU}(N-1)$ transformations we can bring the matrix $A_{1}$ to a standard form with only entries around the diagonal

$$
A_{1}=\left(\begin{array}{lll}
a_{1} \epsilon & & \\
& a_{2} \epsilon & \\
& & \ddots
\end{array}\right), \quad \epsilon=\left(\begin{array}{cc}
0 & 1 \\
-1 & 0
\end{array}\right) .
$$

The F-term matrix $\bar{F}$ is then a diagonal matrix. Given this, when $\mathrm{N}$ is odd, the last row and column of $A_{1}$ are all zero, hence the $\mathrm{F}$-flatness implies that $A_{1}$ is zero entirely. When $N=2 n$ even, the absolute values $\left|a_{i}\right|$ of the eigenvalues of $A_{1}$ are all equal; the corresponding gauge symmetry breaking is $\mathrm{SU}(2 n) \rightarrow \mathrm{Sp}(2 n)$. As can be see in Table 4 this possibility has been used to obtain the bundle model with instanton number $(0,0,0,4)$ from the heterotic orbifold $3 \mathrm{c}$, given in Table 1.

\section{one antisymmetric tensor and one fundamental}

Next consider the situation with one antisymmetric tensor $A=\left(A_{1}, A_{2}\right)$ and a fundamental $\phi=$ $\left(\phi_{1}, \phi_{2}\right)$ of $\mathrm{SU}(N)$. For $N$ is even we find a previous case back in which only the antisymmetric tensor has a VEV. For $N=2 n+1$ a new possibility arises because the fundamental $\phi_{1}$ can precisely be 
non-vanishing in the direction where the anti-symmetric matrix in the skew-diagonal form is totally vanishing:

$$
\phi_{1}=\left(\begin{array}{c}
c \\
0 \\
\vdots
\end{array}\right), \quad A_{1}=\left(\begin{array}{lll}
0 & & \\
& a_{1} \epsilon & \\
& & \ddots
\end{array}\right) .
$$

The $\mathrm{F}$-flatness then requires that the phases $\alpha_{\phi}=\alpha_{A}$ and all entries have equal absolute values: $|c|=\left|a_{i}\right|$. The corresponding symmetry breaking is $\mathrm{SU}(2 \mathrm{n}+1) \rightarrow \mathrm{Sp}(2 \mathrm{n})$. The bundle model with instanton number $(0,0,0,2)$ and line bundle vector $\left(2,0^{15}\right)$, see Table 4 , can be realized in this way from the heterotic orbifold model $3 \mathrm{~b}$ of Table 1.

\section{two antisymmetric tensors (and a fundamental)}

When two antisymmetric tensors $A=\left(A_{1}, A_{2}\right)$ and $B=\left(B_{1}, B_{2}\right)$ take non-vanishing VEVs the F-term reads

$$
\bar{F}=\alpha_{A} A_{1} A_{1}^{\dagger}+\alpha_{B} B_{1} B_{1}^{\dagger} .
$$

Using an $\mathrm{SU}(N-1)$ transformation we can only bring one into the form where all entries except those immediately off the diagonal vanish. Only when both $A_{1}$ and $B_{1}$ are skew-diagonal, the off-diagonal entries of $\bar{F}$ all vanish. We see that for $\mathrm{N}$ is even there are two classes of solutions:

$$
\begin{aligned}
& \alpha_{A}=\alpha_{B}=1: \quad\left|a_{i}\right|^{2}+\left|b_{i}\right|^{2}=r^{2}, \\
& \alpha_{A}=-\alpha_{B}=1:\left|a_{i}\right|^{2}-\left|b_{i}\right|^{2}=r^{2} .
\end{aligned}
$$

When $N$ is odd only the second solution is available for $r=0$.

Depending on whether some of the eigenvalues are equal and non-zero, zero or different, the gauge symmetry breaking varies. These different possibilities are continously connected in the moduli space because they are obtained from varying some of these eigenvalues. For the SU(8) gauge group of heterotic model 3c, see Table 1, the possible unbroken gauge groups range among $\operatorname{Sp}(8), \operatorname{Sp}(6) \times$ $\mathrm{SU}(2), \mathrm{Sp}(4) \times \mathrm{SU}(4)$ and $\mathrm{SU}(2) \times \mathrm{SU}(6)$ are realized as bundle models, see Table 4 . Again the model with the largest unbroken gauge group, $\operatorname{Sp}(8)$, i.e. the model with instanton numbers $(0,0,0,4)$, has another realization using only twisted states. In this case there are many other unbroken gauge groups possible that do not occur in Table 4 as we discuss in the main text.

The twisted and untwisted anti-symmetric tensors in heterotic models $3 \mathrm{~b}$ and $3 \mathrm{c}$ have different $\mathrm{U}(1)$ charges, see Table 1 . This means that alone they cannot achieve both $\mathrm{SU}(\mathrm{N})$ and U(1)-flat configurations; an extra charged field is needed. In model 3c there exists a twisted charged singlet. For model $3 \mathrm{~b}$ we can use one of the two $(\mathbf{1}, \mathbf{5})$ to find the unbroken gauge groups $\mathrm{Sp}(4)$ and $\mathrm{SU}(2)^{2}$. The moduli space of both $\mathrm{SU}(5)$ and $\mathrm{U}(1)$-flat configurations thus combines the results of this and the previous paragraph:

$$
\left|b_{1}\right|^{2}=|c|^{2}-\left|a_{1}\right|^{2}, \quad\left|b_{2}\right|^{2}=|c|^{2}-\left|a_{2}\right|^{2}, \quad|c|^{2}=\frac{5}{2}\left(\left|a_{1}\right|^{2}+\left|a_{2}\right|^{2}\right) .
$$

Therefore generically the surviving gauge group is $\mathrm{SU}(2)^{2}$, however when $\left|a_{1}\right|=\left|a_{2}\right|$ the symmetry is enhanced to $\mathrm{Sp}(4)$. This corresponds to the bundle model with instanton numbers $(0,0,0,2)$ and line bundle vector $\left(2,0^{15}\right)$, see Table 4 . The generic situation describes the model with instanton number $(0,0,0,1)$ and line bundle vector $\left(2,1^{2}, 0^{13}\right)$. 


\section{C.3 $\quad \mathrm{SU}(N) \times \mathrm{SU}(2) \times \mathrm{SU}(2)^{\prime}-$ flatness}

The heterotic model 3e of Table 1 has gauge group $\mathrm{SU}(14) \times \mathrm{SU}(2) \times \mathrm{SU}(2)^{\prime}$. Apart from the two $\mathrm{SU}(2)$ doublets, the twisted spectrum contains a $(\mathbf{1 4}, \mathbf{2}, \mathbf{1})$. Using similar arguments as presented for a single fundamental of $\mathrm{SU}(N)$ one concludes that a VEV for this state alone is impossible. Therefore, combined $\mathrm{SU}(14) \times \mathrm{SU}(2) \times \mathrm{SU}(2)^{\prime}$-flat configurations are only possible, if we give the untwisted $(\mathbf{1 4}, \mathbf{2}, \mathbf{2})$, and the twisted $(\mathbf{1 4}, \mathbf{2}, \mathbf{1})$ and $(\mathbf{1}, \mathbf{1}, \mathbf{2})$ VEV's simultaneously. Denoting the SU(14), SU(2) and SU $(2)^{\prime}$ indices as $a=1, \ldots N, i=1,2$ and $\alpha=1,2$, respectively, these hypermultiplets are $\phi_{a i \alpha}, \psi_{a i}$ and $\chi_{\alpha}$. The F-terms read:

$$
\bar{F}_{N}=\alpha \phi_{a i \alpha} \bar{\phi}_{b i \alpha}+\beta \psi_{a i} \bar{\psi}_{b i}, \quad \bar{F}_{2}=\alpha \phi_{a i \alpha} \bar{\phi}_{a j \alpha}+\beta \psi_{a i} \bar{\psi}_{a j}, \quad \bar{F}_{2}^{\prime}=\alpha \phi_{a i \alpha} \bar{\phi}_{a i \beta}+\gamma \chi_{\alpha} \bar{\chi}_{\beta},
$$

with $\alpha, \beta$ and $\gamma$ the alignment phases. Let $v_{a}$ be an arbitrary non-vanishing $\mathrm{SU}(N)$ fundamental, and let $e_{i}=\delta_{i 1}$ and $\tilde{e}_{i}=\delta_{i 2}$ be the standard basis vectors in two dimensions. When we take $\alpha=\gamma=-\beta$, we can find two flat solutions. The first one has

$$
\phi_{a i \alpha}=v_{a} e_{i} e_{\alpha}, \quad \psi_{a i}=v_{a} e_{i}, \quad \chi_{\alpha}=c \tilde{e}_{\alpha}
$$

with $|c|^{2}=|v|^{2}$ and surviving gauge group $\mathrm{SU}(N-1)$. This is the blowup realization of the bundle

model with instanton numbers $(0,0,0,2)$ and line bundle vector $\frac{1}{2}(\overbrace{1^{2},-1^{2}}, 1^{12})$ of Table 4 . The other solution involves a second $\mathrm{SU}(N)$ fundamental $w_{a}$ which is independent of the first, say $w \cdot v=0$, so that the configuration

$$
\phi_{a i \alpha}=\left(v_{a} e_{i}+w_{a} \tilde{e}_{i}\right) e_{\alpha}, \quad \psi_{a i}=v_{a} e_{i}+w_{a} \tilde{e}_{i}, \quad \chi_{\alpha}=c \tilde{e}_{\alpha}
$$

with $|c|^{2}=|v|^{2}+|w|^{2}$ can be constructed, the unbroken gauge group is then $\mathrm{SU}(N-2)$. This leads to the second bundle model with a spinorial line bundle vector (i.e. with instanton number $(0,0,0,1)$ and line bundle vector $\left.\frac{1}{2}(\overbrace{1,-1}, 1^{12}, 3^{2})\right)$.

\section{C.4 $\mathrm{SO}(M) \times \mathrm{SU}(N)$-flatness}

In the analysis so far we only considered VEV's for representations of $\mathrm{SU}(N)$ groups. As Table 1 models also includes $\mathrm{SO}(M)$, we have to analyze $\mathrm{SO}(M)$-flatness issues as well. The group $\mathrm{SO}(M)$ has antisymmetric generators $T^{m n}=-T^{n m}$, with $m, n=1, \ldots M$. We see from this table that we only need the vector and spinor representations of $\mathrm{SO}(M)$ groups.

In the vector representation of $\mathrm{SO}(M)$ the generators take the form: $\left(T^{m n}\right)_{i j}=\delta_{i}^{m} \delta_{j}^{n}-\delta_{j}^{m} \delta_{i}^{n}$, so that for a contraction with two vectors $\phi$ and $\psi$ we have

$$
(\phi \wedge \psi)^{m n}=\phi^{i} T_{i j}^{m n} \psi^{j}=\phi^{m} \psi^{n}-\phi^{n} \psi^{m} .
$$

Hence, we can efficiently use the index-free formalism of 2 -forms with the wedge product $\wedge$. As a warm up, we first consider flatness for a single hypermultiplet in the vector representation, containing the two complex vectors $\phi_{1}^{i}$ and $\phi_{2}^{i}$. We have the following $\bar{F}$ and $D$-term two-forms

$$
\bar{F}=\phi_{1} \wedge \phi_{2}, \quad D=\phi_{1} \wedge \bar{\phi}_{1}-\bar{\phi}_{2} \wedge \phi_{2} .
$$


As usual we assume internal alignment so that the $\mathrm{D}$-term vanish automatically and the $\mathrm{F}$-term becomes

$$
\bar{F}=\alpha_{\phi} \phi_{1} \wedge \bar{\phi}_{1}=\alpha_{\phi} \operatorname{Re} \wedge \operatorname{Im}=0
$$

where we split $\phi_{1}$ into its the real and imaginary parts $\phi_{1}=R e+i I m$. Hence, F-flatness is satisfied when $R e= \pm I m, R e=0$ or $I m=0$, but in any case the gauge group is broken to $\mathrm{SO}(M-1)$.

According to Table 1 model 3b has a $\mathrm{SO}(22)$ vector, so SO-flatness can be achieved in the way just described. But this twisted state also carries U(1) charge and hence at least another charged field needs to take a non-vanishing VEV. Since all the other states are also charged under SU(5) we find complicated VEV configurations.

\section{bi-fundamental and vectors of both groups}

The first configuration of this type corresponds to the bundle model with instanton number $(0,0,0,1)$ and line bundle vector $(\overbrace{1,-1}^{1}, 1^{4}, 0^{10})$ given in 4 : Its non-vanishing fields are bi-fundamental $\phi=$ $\left(\phi_{1}, \phi_{2}\right)$, the SO-vector $\psi=\left(\psi_{1}, \psi_{2}\right)$ and the SU-fundamental $\chi=\left(\chi_{1}, \chi_{2}\right)$, and their internal alignment phases are $\alpha, \beta$ and $\gamma$, respectively. Their VEVs are assigned as

$$
\left(\phi_{1}\right)_{a i}=v_{a} e_{i}, \quad\left(\psi_{1}\right)_{a}=w_{a}, \quad\left(\chi_{1}\right)_{i}=c e_{i}
$$

where $v$ and $w$ are two real vectors that are perpendicular to ensure SO-flatness, and $c$ a complex constant. The phases are chosen as $\alpha=-\beta=-\gamma=1$, then $|c|=v^{2}$ for SU-flatness, and $w^{2}$ is adjusted to also have $\mathrm{U}(1)$ flatness. If we instead take $w=v$, still all flatness conditions can be fulfilled but we end up with the bundle model with instanton number $(1,0,0,0)$ and the non-overlapping line bundle vector $\left(1^{4}, 0^{12}\right)$.

\section{bi-fundamental, SO-vector and SU-antisymmetric tensor}

Another configuration employs the VEV's of the bi-fundamental, one SU-antisymmetric tensor and an SO-vector. When their VEV assignments are given by

$$
\left(\phi_{1}\right)_{a}=v_{a}, \quad\left(\psi_{1}\right)_{a i}=v_{a} e_{i}, \quad A_{1}=\left(\begin{array}{ccc}
a \epsilon & & \\
& a \epsilon & \\
& & 0
\end{array}\right),
$$

flatness is achieved provided that $|c|=|v|$. The resulting unbroken gauge group reads $\mathrm{SO}(21) \times \operatorname{Sp}(4)$, hence this gives the blowup realization of the bundle model with instanton number $(1,0,0,2)$ that has no additional line bundle embedding.

\section{bi-fundamental, SO-vector and two SU-antisymmetric tensors}

A third type of configurations combines VEV's of the bi-fundamental, two SU-antisymmetric tensors, and the SO-vector. Their VEV's are

$$
\left(\phi_{1}\right)_{a i}=v_{a} e_{i}, \quad\left(\psi_{1}\right)_{a}=w_{a}, \quad A_{1}=\left(\begin{array}{ccc}
a \epsilon & & \\
& 0 & \\
& & 0
\end{array}\right), \quad B_{1}=\left(\begin{array}{lll}
0 & & \\
& b \epsilon & \\
& & 0
\end{array}\right),
$$


and lead to the symmetry breaking from $\mathrm{SO}(22) \times \mathrm{SU}(5)$ to $\mathrm{SO}(20) \times \mathrm{SU}(2)^{2}$ provided that $v$ and $w$ are perpendicular. This corresponds to the bundle model with instanton number $(0,0,0,2)$ and line

bundle vector $(\overbrace{1,-1}, 1^{2}, 0^{12})$ in Table 4 . When the two vectors $v$ and $w$ are equal, the gauge symmetry is only broken to $\mathrm{SO}(21) \times \mathrm{SU}(2)^{2}$, i.e. we recover the bundle model with instanton number $(1,0,0,1)$ and non-overlapping line bundle vector $\left(1^{2}, 0^{14}\right)$.

\section{two bi-fundamentals}

In the final configuration we consider, there are two bi-fundamentals. We can view the components of the bi-fundamentals as $\mathrm{SO}$-vectors $a, b, c$ and $d$

$$
\sigma_{1}=\left(\begin{array}{ll}
a & b
\end{array}\right), \quad \psi_{1}=\left(\begin{array}{ll}
c & d
\end{array}\right) .
$$

The SO-flatness is fulfilled when these vectors are all real. SU-flatness gives the conditions

$$
\bar{a} b \pm \bar{c} d=0, \quad|a|^{2} \pm|c|^{2}=|b|^{2} \pm|d|^{2},
$$

where the \pm -sign distinguishes between two possible alignments. By taking the vectors perpendicular when they are not proportional, one solves the first equation trivially. With this class of VEV configurations various bundle models are obtained: When all four vectors are perpendicular we end up with gauge group $\mathrm{SO}(24)$, i.e. the bundle model with instanton number $(0,0,0,2)$ and fully overlapping line

bundle vector $(\overbrace{1^{2},-1^{2}}, 0^{12})$. With three non-vanishing vectors with $|b|^{2}=|a|^{2}+|c|^{2}$ and $d=0$ we have the gauge group $S O(25)$ : the bundle model with $n=(1,0,0,1)$ and $V=(\overbrace{1,-1}, 0^{14})$. Finally when we align all four vectors, the gauge group is $\mathrm{SO}(27)$; the bundle model $n=(1,0,0,0)$ and $V=\left(2,0^{15}\right)$ is found.

\section{References}

[1] A. E. Faraggi, D. V. Nanopoulos, and K.-j. Yuan "A standard like model in the 4d free fermionic string formulation" Nucl. Phys. B335 (1990) 347.

[2] R. Blumenhagen, B. Kors, D. Lust, and T. Ott "The Standard Model from stable intersecting brane world orbifolds" Nucl. Phys. B616 (2001) 3-33 [hep-th/0107138].

[3] M. Cvetic, G. Shiu, and A. M. Uranga "Chiral four-dimensional N = 1 supersymmetric type IIA orientifolds from intersecting D6-branes" Nucl. Phys. B615 (2001) 3-32 [hep-th/0107166].

[4] T. P. T. Dijkstra, L. R. Huiszoon, and A. N. Schellekens "Supersymmetric Standard Model spectra from RCFT orientifolds" Nucl. Phys. B710 (2005) 3-57 [hep-th/0411129].

[5] T. P. T. Dijkstra, L. R. Huiszoon, and A. N. Schellekens "Chiral supersymmetric Standard Model spectra from orientifolds of Gepner models" Phys. Lett. B609 (2005) 408-417 [hep-th/0403196].

[6] L. Dixon, J. A. Harvey, C. Vafa, and E. Witten "Strings on orbifolds" Nucl. Phys. B261 (1985) 678-686.

[7] L. J. Dixon, J. A. Harvey, C. Vafa, and E. Witten "Strings on orbifolds. 2" Nucl. Phys. B274 (1986) $285-314$.

[8] L. E. Ibanez, H. P. Nilles, and F. Quevedo "Orbifolds and Wilson lines" Phys. Lett. B187 (1987) 25-32.

[9] W. Buchmuller, K. Hamaguchi, O. Lebedev, and M. Ratz "Supersymmetric Standard Model from the heterotic string" Phys. Rev. Lett. 96 (2006) 121602 [hep-ph/0511035]. 
[10] W. Buchmuller, K. Hamaguchi, O. Lebedev, and M. Ratz "Supersymmetric Standard Model from the heterotic string. II" Nucl. Phys. B785 (2007) 149-209 [hep-th/0606187].

[11] O. Lebedev et al. "A mini-landscape of exact MSSM spectra in heterotic orbifolds" Phys. Lett. B645 (2007) 88-94 [hep-th/0611095].

[12] G. Altarelli and F. Feruglio "SU(5) grand unification in extra dimensions and proton decay" Phys. Lett. B511 (2001) 257-264 [hep-ph/0102301].

[13] T. Kobayashi, S. Raby, and R.-J. Zhang "Searching for realistic 4d string models with a Pati-Salam symmetry: Orbifold grand unified theories from heterotic string compactification on a Z(6) orbifold" Nucl. Phys. B704 (2005) 3-55 [hep-ph/0409098].

[14] S. Forste, H. P. Nilles, P. K. S. Vaudrevange, and A. Wingerter "Heterotic brane world" Phys. Rev. D70 (2004) 106008 [hep-th/0406208].

[15] A. Hebecker and M. Trapletti "Gauge unification in highly anisotropic string compactifications" Nucl. Phys. B713 (2005) 173-203 [hep-th/0411131].

[16] J. E. Kim and B. Kyae "Flipped SU(5) from Z(12-I) orbifold with Wilson line" Nucl. Phys. B770 (2007) 47-82 [hep-th/0608086].

[17] S. Donalson "Anti-self-dual Yang-Mills connections over complex algebraic surfaces and stable vector bundles" Proc. Londan Math. Soc. 50 (1985) 1-26.

[18] K. Uhlenbeck and S. Yau "On the existence of Hermitian-Yang-Mills connections in stable vector bundles" Comm. Pure and Appl. Math. 19 (1986) 257-293.

[19] V. Braun, Y.-H. He, B. A. Ovrut, and T. Pantev "A heterotic standard model" Phys. Lett. B618 (2005) 252-258 [hep-th/0501070].

[20] V. Braun, Y.-H. He, B. A. Ovrut, and T. Pantev "A standard model from the E(8) x E(8) heterotic superstring" JHEP 06 (2005) 039 [hep-th/0502155].

[21] R. Blumenhagen, G. Honecker, and T. Weigand "Non-Abelian brane worlds: The heterotic string story" JHEP 10 (2005) 086 [hep-th/0510049].

[22] V. Bouchard and R. Donagi "An SU(5) heterotic standard model" Phys. Lett. B633 (2006) 783-791 [hep-th/0512149].

[23] R. Blumenhagen, S. Moster, and T. Weigand "Heterotic GUT and Standard Model vacua from simply connected Calabi-Yau manifolds" Nucl. Phys. B751 (2006) 186-221 [hep-th/0603015].

[24] G. Honecker and M. Trapletti "Merging heterotic orbifolds and K3 compactifications with line bundles" JHEP 01 (2007) 051 [hep-th/0612030].

[25] S. Groot Nibbelink, M. Trapletti, and M. Walter "Resolutions of $\mathrm{C}^{* *} \mathrm{n} / \mathrm{Z}(\mathrm{n})$ orbifolds, their U(1) bundles, and applications to string model building" JHEP 03 (2007) 035 [hep-th/0701227].

[26] O. J. Ganor and J. Sonnenschein "On the strong coupling dynamics of heterotic string theory on C**3/Z(3)" JHEP 05 (2002) 018 [hep-th/0202206].

[27] S. Groot Nibbelink, H. P. Nilles, and M. Trapletti "Multiple anomalous U(1)s in heterotic blow-ups" Phys. Lett. B652 (2007) 124-127 [hep-th/0703211].

[28] S. Groot Nibbelink, D. Klevers, F. Ploger, M. Trapletti, and P. K. S. Vaudrevange "Compact heterotic orbifolds in blow-up" JHEP 04 (2008) 060 [0802.2809].

[29] J. Erler and A. Klemm "Comment on the generation number in orbifold compactifications" Commun. Math. Phys. 153 (1993) 579-604 [hep-th/9207111]. 
[30] P. S. Aspinwall "Resolution of orbifold singularities in string theory" [hep-th/9403123].

[31] D. Lust, S. Reffert, E. Scheidegger, and S. Stieberger "Resolved toroidal orbifolds and their orientifolds" [hep-th/0609014].

[32] S. Groot Nibbelink, T.-W. Ha, and M. Trapletti "Toric Resolutions of Heterotic Orbifolds" Phys. Rev. D77 (2008) 026002 [0707.1597].

[33] T. Eguchi and A. J. Hanson "Asymptotically Flat Selfdual Solutions to Euclidean Gravity" Phys. Lett. B74 (1978) 249.

[34] G. W. Gibbons and S. W. Hawking "Gravitational Multi - Instantons" Phys. Lett. B78 (1978) 430.

[35] T. Eguchi, P. B. Gilkey, and A. J. Hanson "Gravitation, Gauge Theories and Differential Geometry" Phys. Rept. 66 (1980) 213.

[36] M. R. Douglas and G. W. Moore "D-branes, Quivers, and ALE Instantons" [hep-th/9603167].

[37] M. Bianchi, F. Fucito, G. Rossi, and M. Martellini "Explicit Construction of Yang-Mills Instantons on ALE Spaces" Nucl. Phys. B473 (1996) 367-404 [hep-th/9601162].

[38] J. Conrad Orbifolds and Kaluza-Klein-Monopoles in Heterotic E8xE8 String Theory Preserving Eight Supercharges. PhD thesis Bonn University 2001. http://thp.uni-bonn.de/nilles/db/thesis/conrad.pdf.

[39] T. Eguchi and A. J. Hanson "Selfdual Solutions to Euclidean Gravity" Ann. Phys. 120 (1979) 82.

[40] A. Sen "Dynamics of multiple Kaluza-Klein monopoles in M and string theory" Adv. Theor. Math. Phys. 1 (1998) 115-126 [hep-th/9707042].

[41] P. J. Ruback "The motion of Kaluza-Klein monopoles" Commun. Math. Phys. 107 (1986) 93-102.

[42] G. 't Hooft "Magnetic monopoles in unified gauge theories" Nucl. Phys. B79 (1974) 276-284.

[43] M. Nakahara "Geometry, topology and physics". Bristol, UK: Hilger (1990) 505 p. (Graduate student series in physics).

[44] M. F. Atiyah, N. J. Hitchin, V. G. Drinfeld, and Y. I. Manin "Construction of instantons" Phys. Lett. A65 (1978) 185-187.

[45] N. Dorey, T. J. Hollowood, V. V. Khoze, and M. P. Mattis "The calculus of many instantons" Phys. Rept. 371 (2002) 231-459 [hep-th/0206063].

[46] P. B. Kronheimer "The Construction of ALE spaces as hyperKahler quotients" J. Diff. Geom. 29 (1989) 665-683.

[47] P. B. Kronheimer and H. Nakajima "Yang-Mills instantons on ALE gravitational instantons" Math. Ann. 288 (1990) 263-307.

[48] P. B. Kronheimer and H. Nakajima "Moduli spaces of anti-self-dual connections on ALE gravitational instantons" Invent. Math. 102 (1990) 267-303.

[49] F. Fucito, J. F. Morales, and R. Poghossian "Multi instanton calculus on ALE spaces" Nucl. Phys. B703 (2004) 518-536 [hep-th/0406243].

[50] F. Wilczek "Inequivalent embeddings of SU(2) and instanton interactions" Phys. Lett. B65 (1976) $160-162$.

[51] S. Vandoren and P. van Nieuwenhuizen "Lectures on instantons" [0802.1862].

[52] W. Fulton Introduction to Toric Varieties. Princeton University Press 1993.

[53] T. Oda Convex Bodies and Algebraic Geometry: An Introduction to the Theory of Toric Varieties. Springer 1988. 
[54] V. Bouchard "Lectures on complex geometry, Calabi-Yau manifolds and toric geometry" [hep-th/0702063].

[55] G. Aldazabal, A. Font, L. E. Ibanez, A. M. Uranga, and G. Violero "Non-perturbative heterotic D = 6,4, N = 1 orbifold vacua" Nucl. Phys. B519 (1998) 239-281 [hep-th/9706158].

[56] L. E. Ibanez and A. M. Uranga "D =6, N =1 string vacua and duality" [hep-th/9707075].

[57] E. Witten "Global gravitational anomalies" Commun. Math. Phys. 100 (1985) 197.

[58] D. S. Freed "Determinants, torsion, and strings" Commun. Math. Phys. 107 (1986) 483-513.

[59] G. Honecker "Massive U(1)s and heterotic five-branes on K3" Nucl. Phys. B748 (2006) 126-148 [hep-th/0602101].

[60] R. Slansky "Group theory for unified model building" Phys. Rept. 79 (1981) 1-128.

[61] M. B. Green, J. H. Schwarz, and P. C. West "Anomaly free chiral theories in six-dimensions" Nucl. Phys. B254 (1985) 327-348.

[62] J. Erler "Anomaly cancellation in six-dimensions" J. Math. Phys. 35 (1994) 1819-1833 [hep-th/9304104].

[63] M. R. Douglas, B. R. Greene, and D. R. Morrison "Orbifold resolution by D-branes" Nucl. Phys. B506 (1997) 84-106 [hep-th/9704151].

[64] N. Seiberg and E. Witten "Comments on String Dynamics in Six Dimensions" Nucl. Phys. B471 (1996) 121-134 [hep-th/9603003].

[65] M. J. Duff, R. Minasian, and E. Witten "Evidence for Heterotic/Heterotic Duality" Nucl. Phys. B465 (1996) 413-438 [hep-th/9601036].

[66] E. Witten "Some properties of O(32) superstrings" Phys. Lett. B149 (1984) 351-356.

[67] H. Georgi "Lie algebras in particle physics. from isospin to unified theories" Front. Phys. 54 (1982) 1-255. 